\title{
OPEN De novo transcriptome assembly and comparative transcriptomic analysis provide molecular insights into low temperature stress response of Canarium album
}

\author{
Ruilian Lai ${ }^{1}$, Xin Feng ${ }^{1}$, Jin Chen ${ }^{1}$, Yongyan Zhang ${ }^{2}$, Xiaoxia Wei ${ }^{1}$, Yiting Chen ${ }^{1}$,
} Chunzhen Cheng ${ }^{2 \bowtie}$ \& Rujian Wu ${ }^{1 凶}$

A de novo transcriptome analysis was performed in C. album, a temperature sensitive fruit tree in China, after treatment with varied temperatures. A total number of 168,385 transcripts were assembled, comprising of 109,439 unigenes, of which 70,530 were successfully annotated. Compared with control check group $(\mathrm{CK})$, which was treated under $25^{\circ} \mathrm{C}$, the chilling stress $\left(4^{\circ} \mathrm{C}\right)$ treated group (CT), showed about 2810 up-regulated and 2567 down-regulated genes. Whereas, group treated under freezing $\left(-3^{\circ} \mathrm{C}\right)$ stress (FT) showed an up-regulation and a down-regulation of 1748 and 1459 genes, respectively. GO classification analysis revealed that DEGs related to metabolic processes, single-organism metabolic process, and catalytic activity are significantly enriched in both CT and FT conditions. KEGG pathway enrichment analysis for both CT and FT treatments showed an enrichment of genes encoding or related to glycine/serine and threonine metabolism, alpha-linolenic acid metabolism, carotenoid biosynthesis, photosynthesis-antenna proteins, and circadian rhythm. However, genes related to photosynthesis, carbon fixation in photosynthetic organisms, glutathione metabolism, pyruvate metabolism, nicotinate and nicotinamide metabolism were specifically enriched in CT condition. Nevertheless, FT treatment induced genes related to plant-pathogen interaction, linoleic acid metabolism, plant hormone signal transduction and pentose phosphate pathway. Many of the genes involved in plant hormone signal transduction showed significantly different expression in both FT and CT conditions. However, the change was more evident in FT. Here we present the first of the reports for a de novo transcriptomic analysis in C. album, suggesting that the plant shows differential responses in chilling and freezing temperatures, where the hormone signaling and transduction contribute greatly to FT responses. Our study thus paves way for future research regarding functions of these potentially identified genes.

Low temperature (LT) limits the geographical distribution and cultivation of many crops because of plant growth inhibition, substantial reduction in production, and even plant death ${ }^{1}$. Generally, it is divided into chilling injury $\left(0-15^{\circ} \mathrm{C}\right)$ and freezing injury $\left(<0^{\circ} \mathrm{C}\right)$. Plants originating from temperate regions undergo adaptive resistive acclimation at low, non-freezing temperatures or temperature slightly below freezing point, but severe damages can still be caused under extremely critical LT. However, plants originating from tropical and subtropical regions are usually LT-sensitive. Under LT stress the cell structure, membrane composition, antioxidant component and gene transcription of plant changes significantly causing physiological disorders related to water status, mineral nutrition, photosynthesis, respiratory rate, and metabolism etc. ${ }^{2}$. Cold tolerance in plants is usually controlled by two categories of genes: the functional or structural genes ${ }^{3,4}$ encoding functional proteins that directly respond to LT stress and the regulatory genes ${ }^{5}$ that code for protein kinases and transcription factors regulating the expression or signal transduction of downstream targets of LT responsive genes.

${ }^{1}$ Fruit Research Institute, Fujian Academy of Agricultural Sciences, Fuzhou 350013, China. ${ }^{2}$ College of Horticulture, Fujian Agriculture and Forestry University, Fuzhou 350002, China. ${ }^{\square}$ email: Id0532cheng@126.com; wurujian@126.com 


\begin{tabular}{|l|l|l|l|l|l|l|l|}
\hline Sample & Raw reads number & Clean reads number (\%) & Clean bases & Error rate (\%) & $\mathbf{Q}_{\mathbf{2 0}}(\%)$ & $\mathbf{Q}_{\mathbf{3 0}}(\%)$ & GC content (\%) \\
\hline CK & $44,734,290$ & $41,844,672(94.40)$ & $6.33 \mathrm{G}$ & 0.02 & 96.42 & 90.85 & 43.11 \\
\hline CT & $44,448,624$ & $42,409,426(94.14)$ & $6.28 \mathrm{G}$ & 0.02 & 96.52 & 91.00 & 42.78 \\
\hline FT & $44,987,308$ & $42,230,274(94.27)$ & $6.36 \mathrm{G}$ & 0.02 & 96.46 & 90.87 & 43.17 \\
\hline
\end{tabular}

Table 1. RNA-Seq results. $\mathrm{Q}_{20} / \mathrm{Q}_{30}$ mean the rate of bases whose $p$-values were more than $20 / 30$.

RNA sequencing technology (RNA-Seq) is widely helpful in discovering the molecular mechanisms involved in plant stress responses including changes to metabolism and the relationship between host energy metabolism, signal transduction and various defense responses ${ }^{6}$. It utilizes high throughput analysis that covers a wide range of RNAs and provides high precision too. For many minor crops, the information related to unigenes or expressed sequence tags (ESTs) is very limited, which greatly hinders the research advancement in their case. RNA-Seq, however, being reference genome independent, counts for an economic, effective, and widely used research method for obtaining transcriptomic data for any organism. Recently, with the application of RNASeq, research involving transcriptome analysis had been carried out on many plants and great progress has been made in interpreting the LT response mechanisms, screening of key regulatory genes and construction of metabolism regulation network. Many putative genes contributing to the cold stress response in Rumex patientia, including the members of $M Y B, A P 2 / E R F, C B F, Z n f, b Z I P, N A C$ and $C O R$ gene families have been identified ${ }^{7}$. Transcriptional regulation, molecular transport and signal transduction pathways, involved in the adaptation to low temperatures in Saussurea involucrate have also been studied using de novo RNA-Seq ${ }^{8}$. Transcription factors like $A R R-B, b H L H, E R F, M Y B$ and $W R K Y$ etc. are believed to be specifically involved in cold-response in Hevea brasiliensis ${ }^{5}$. Moreover, RNA-Seq is successfully been used in discovering LT stress responses in crops like Oryza sativa, Taxus chinensis, and Hordeum vuglare ${ }^{9-11}$.

Canarium album, an evergreen and high nutritional fruit tree, is mainly distributed and cultivated in Fujian, Guangdong, Zhejiang, Guangxi, Sichuan and Chongqing provinces of China. It is also cultivated in a small numbers in north-central Vietnam, Japan and Malay Peninsula. It has great amounts of tannin, polysaccharide, and petroleum ether ${ }^{12-14}$, that show great antioxidant activities. Gallic acid, produced by C. album, has been proved to have radical scavenging capabilities ${ }^{15}$ and the ethyl acetate and ellagic acid, found in it, can be used for anti-HIV and anti-hepatitis B virus remedies ${ }^{16,17}$. Moreover, C. album is also a source of caffeic acid N-nonyl ester showing good anti-bacterial characteristics ${ }^{18}$.

China has a huge market for freshly available and edible fruits of C. album. However, the C. album tree is quite sensitive to LT. $-3^{\circ} \mathrm{C}$ is considered to be the most critical low temperature that C. album can bear ${ }^{19}$. The main $C$. album production areas in China, such as Shanghang, Minqing, Minhou and Youxi counties in Fujian province and Pingyang and Rui'an counties in Zhejiang province, suffer damages from large-scale low-temperature freezing injury in C. album trees that also cause even the next year's output to plummet ${ }^{16}$. Therefore, LT stress has become one of the most important limiting factors restricting the stable development and expansion of C. album fruit industry. Hence, development of LT-resistant cultivation technology and selection of LT-resistant rootstock are of utmost importance. However, most of the recent studies on C. album were focused on the medicinal and chemical constituent excavation and pharmacological activity identification, very few reports concentrated on LT stress responses per say. Most unfortunately, no reference genome and transcriptome data has been published yet. To discover the LT response mechanisms of C. album, we first assembled the de novo transcriptome and then compared the transcriptomic differences under $-3{ }^{\circ} \mathrm{C}$ (freezing stress treatment, FT), $4^{\circ} \mathrm{C}$ (chilling stress treatment, CT) and $25^{\circ} \mathrm{C}$ (control check, CK) treatment conditions. Results from our study provide basis for the gene function analysis and are helpful for uncovering the LT response mechanisms in C. album.

\section{Results}

RNA-Seq and transcriptome assembly. As shown in Table 1, the $\mathrm{Q}_{20}$ and $\mathrm{Q}_{30}$ value of the three cDNA libraries were $>96 \%$ and $90 \%$, respectively. The error rate for bases was below $0.02 \%$ and the $\mathrm{G} / \mathrm{C}$ contents were around $43 \%$. A total of 44,734,290, 44,448,624 and 44,987,308 original raw reads were obtained for CK, CT and FT, respectively. After removing low quality reads, $94.40 \%(6.33 \mathrm{G}), 94.14 \%(6.28 \mathrm{G})$ and $94.27 \%(6.36 \mathrm{G})$ reads were used for further analysis for CK, CT and FT, respectively.

De novo transcriptome assembly of C. album highlighted 168,385 transcripts, whose $\mathrm{N}_{50}$ and $\mathrm{N}_{90}$ values were $2036 \mathrm{bp}$ and $369 \mathrm{bp}$, respectively. The longest, the shortest and the average length of transcripts were 19,220 bp, $201 \mathrm{bp}$ and $1086 \mathrm{bp}$, respectively. A total of 109,439 unigenes with an average length of $1517 \mathrm{bp}$ were acquired. The $\mathrm{N}_{50}$ and $\mathrm{N}_{90}$ of these unigenes was $2543 \mathrm{bp}$ and $648 \mathrm{bp}$, respectively.

Unigene annotation. The unigenes of C. album were annotated by using Nr, Nt, Pfam, KOG, Swiss-Prot, KEGG and GO databases (Table 2). Results showed that 70,530 unigenes, that accounted for almost $64.44 \%$ of the total number of unigenes, were annotated in at least one database. The $\mathrm{Nr}$ database gave the largest number of unigene annotations (65,274; 59.64\%), while those in KEGG database got the least annotations $(25,196$; $23.02 \%)$. In addition, $11,284(10.31 \%)$ unigenes were annotated in all the databases. The largest distribution of annotation similarity ranged from 60 to $80 \%$ and $80-95 \%$, accounting for $44.60 \%$ and $42.40 \%$, respectively (Fig. 1a). According to RNA-Seq alignment results, Citrus sinensis, C. clementina and Theobroma cacao were the ones having the highest similarity to C. album (Fig. 1b). 


\begin{tabular}{|l|l|l|}
\hline Item & Number of unigenes & Percentage (\%) \\
\hline Annotated in Nr & 65,274 & 59.64 \\
\hline Annotated in Nt & 52,605 & 48.06 \\
\hline Annotated in KEGG & 25,196 & 23.02 \\
\hline Annotated in Swiss-prot & 50,854 & 46.46 \\
\hline Annotated in Pfam & 49,695 & 45.40 \\
\hline Annotated in GO & 50,034 & 45.71 \\
\hline Annotated in KOG & 18,653 & 17.04 \\
\hline Annotated in all databases & 11,284 & 10.31 \\
\hline Annotated in at least one database & 70,530 & 64.44 \\
\hline Total annotation unigenes & 109,439 & 100 \\
\hline
\end{tabular}

Table 2. Annotation results showing the total number of unigenes and their respective percent share as compared to total number of unigenes.

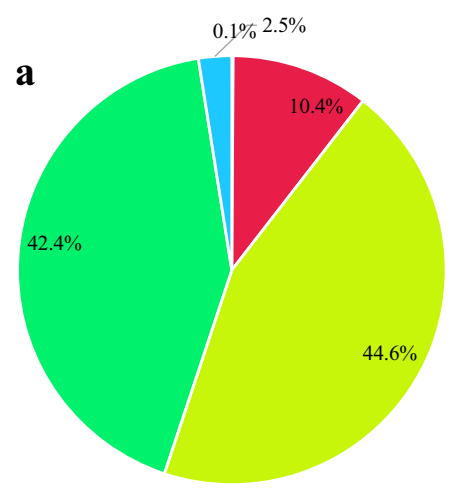

- $18 \%-40 \% \square+40 \%-60 \% \square 60 \%-80 \% \square 80 \%-95 \%-95 \%-100 \%$

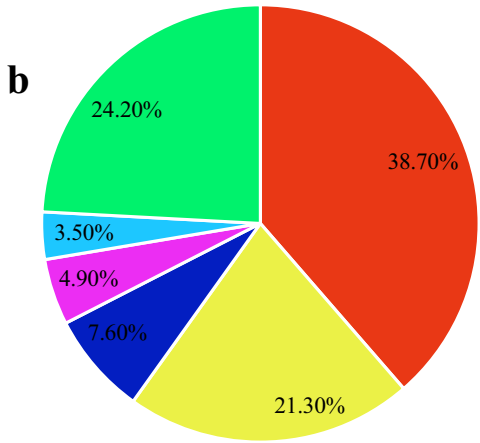

- Citrus sinensis $\quad$ Citrus clementina $\square$ Theobroma cacao - Vitis vinifera $\square$ Jatropha curcas $\square$ other

Figure 1. Similarity distribution and species classification of the annotated unigenes. (a) The pie-chart shows the similarity distribution of annotated unigenes in the mentioned databases. (b) Pie-chart showing the similarity of C. album with other species according to annotated unigenes.

KOG gene function classification of the $C$. album unigenes. The unigenes annotated by KOG were classified into 25 categories (Fig. 2). The proportion of unigenes in general function prediction was the highest with value up to $12.17 \%$ of the total unigenes, followed by posttranslational modification, protein turnover, chaperones category (11.30\%), translation, ribosomal structure and biogenesis $(8.02 \%)$ and RNA processing and modification $(7.23 \%)$ categories. Number of unigenes related to cell mobility were the least in number $(0.06 \%)$ along with unigenes involved in defense mechanisms $(0.07 \%)$.

Gene ontology (GO) analysis of $C$. album unigenes. GO analysis classified the unigenes from $C$. album in three categories, namely, cellular component (CC), molecular function (MF) and biological process (BP) (Fig. 3). 65.73\% of the unigenes were classified into 25 different sub-categories of BP, $19.62 \%$ of the unigenes into 19 sub-categories of CC, and $14.65 \%$ into 10 sub-categories of MF. Further upon BP analysis we found that majority (top three) of the unigenes were related to cellular, metabolic and single-organism processes, while those for behavior, cell aggregation, growth and rhythmic process were very few in number. In CC, unigenes related to cell and cell parts had the largest share, whereas extracellular matrix component, synapse and synapse part had only a few. The genes related to binding and catalytic activity accounted for the largest proportions in $\mathrm{MF}$ and the ones involved in metallochaperone activity comprised a very small proportion.

KEGG classification of the $C$. album unigenes. The unigenes identified in C. album were annotated in 130 KEGG metabolism pathways that belonged to 5 main categories, including those for cellular processes, environmental information processing, genetic information processing, metabolism, and organismal systems (Fig. 4). The greatest number of annotated unigenes were related to metabolism, followed by those involved in genetic information processing. The least number of unigenes belonged to environmental information processing. In the second level of pathway enrichment, the highest number of unigenes were those that are involved in translation and carbohydrate metabolism and the ones involved in membrane transport were the lowest. The top 5 metabolic pathways, based on the number of unigenes involved, were for carbon metabolism, plant-pathogen interaction, ribosome function, amino acid biosynthesis and RNA transport. 


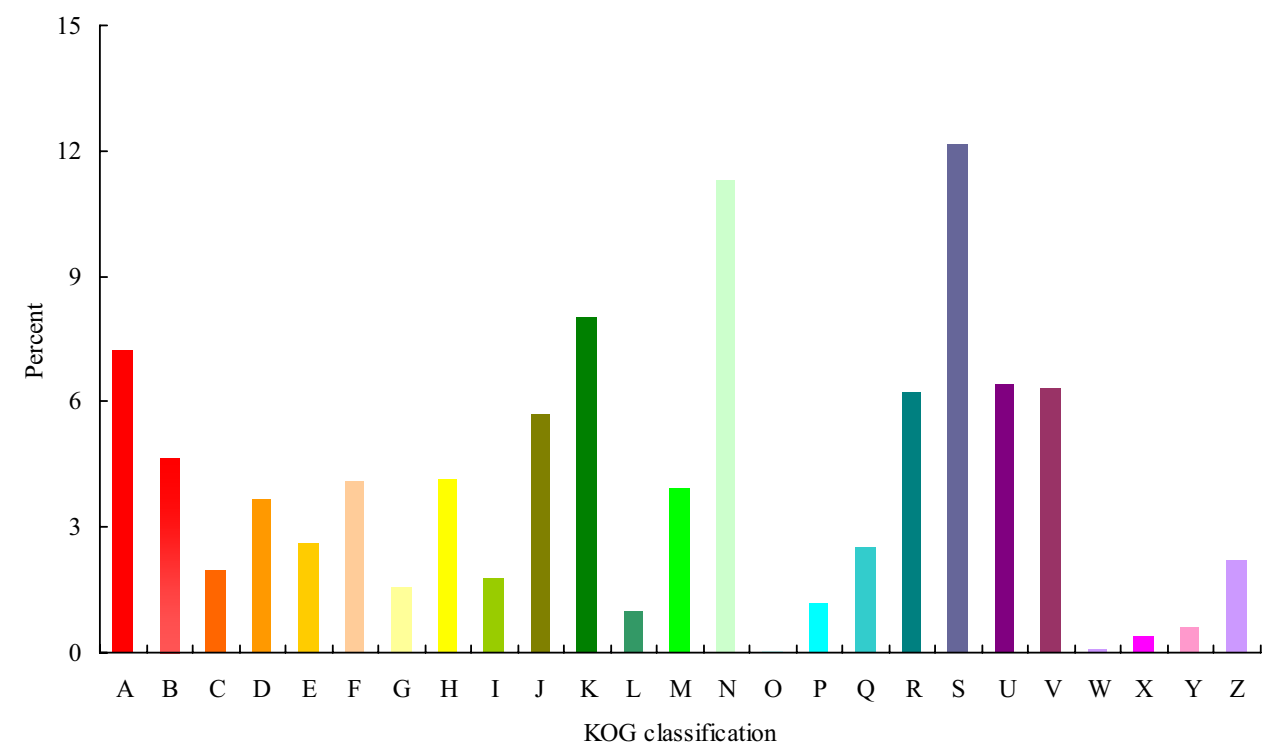

Figure 2. KOG gene function classification of the C. album unigenes. (A) RNA processing and modification, (B) Energy production and conversion, (C) Chromatin structure and dynamics, (D) Amino acid transport and metabolism, (E) Cell cycle control, cell division, chromosome partitioning, (F) Carbohydrate transport and metabolism, (G) Nucleotide transport and metabolism, (H) Lipid transport and metabolism, (I) Coenzyme transport and metabolism, (J) Transcription, (K) Translation, ribosomal structure and biogenesis, (L) Cell wall/membrane/envelope biogenesis, (M) Replication, recombination and repair, (N) Posttranslational modification, protein turnover, chaperones, $(\mathbf{O})$ Cell motility, (P) Secondary metabolites biosynthesis, transport and catabolism, (Q) Inorganic ion transport and metabolism, (R) Function unknown, (S) General function prediction only, (T) Intracellular trafficking, secretion, and vesicular transport, (U) Signal transduction mechanisms, (V) Extracellular structures, (W) Defense mechanisms, (Y) Nuclear structure, (Z) Cytoskeleton.

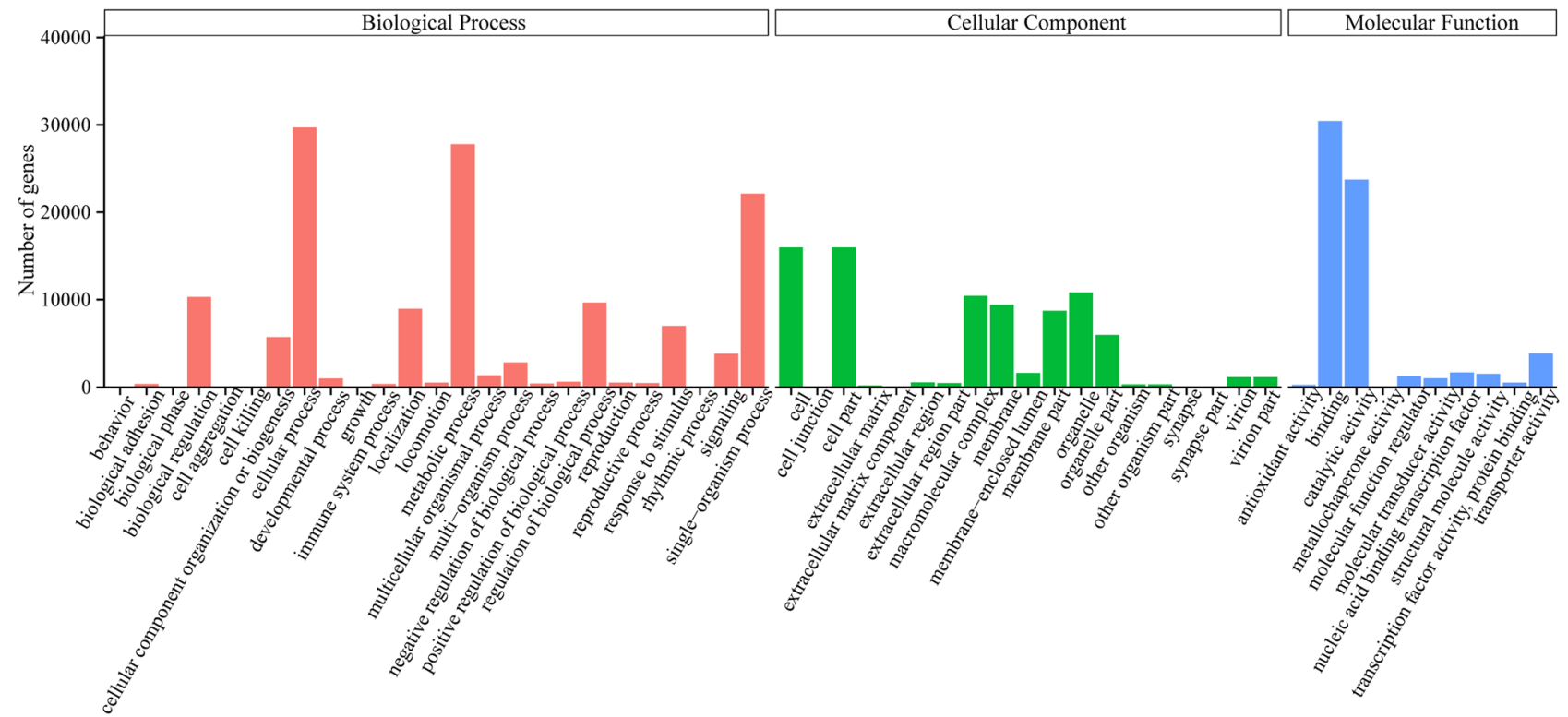

Figure 3. GO gene function classification of C. album unigenes. The biological process, the cellular component and the molecular function were shown in red, green and blue, respectively.

Identification of differentially expressed genes (DEGs) under low temperature treatments. Compared with CK, CT showed 2810 and 2567 genes that were upregulated and downregulated, respectively, while FT resulted in 1748 upregulated and 1459 downregulated ones (Fig. 5). Among these DEGs, 1092 up-regulated and 1148 down-regulated ones were commonly found in both CT and FT (Fig. 6), indicating that they contribute to both chilling and freezing responses in C. album. 


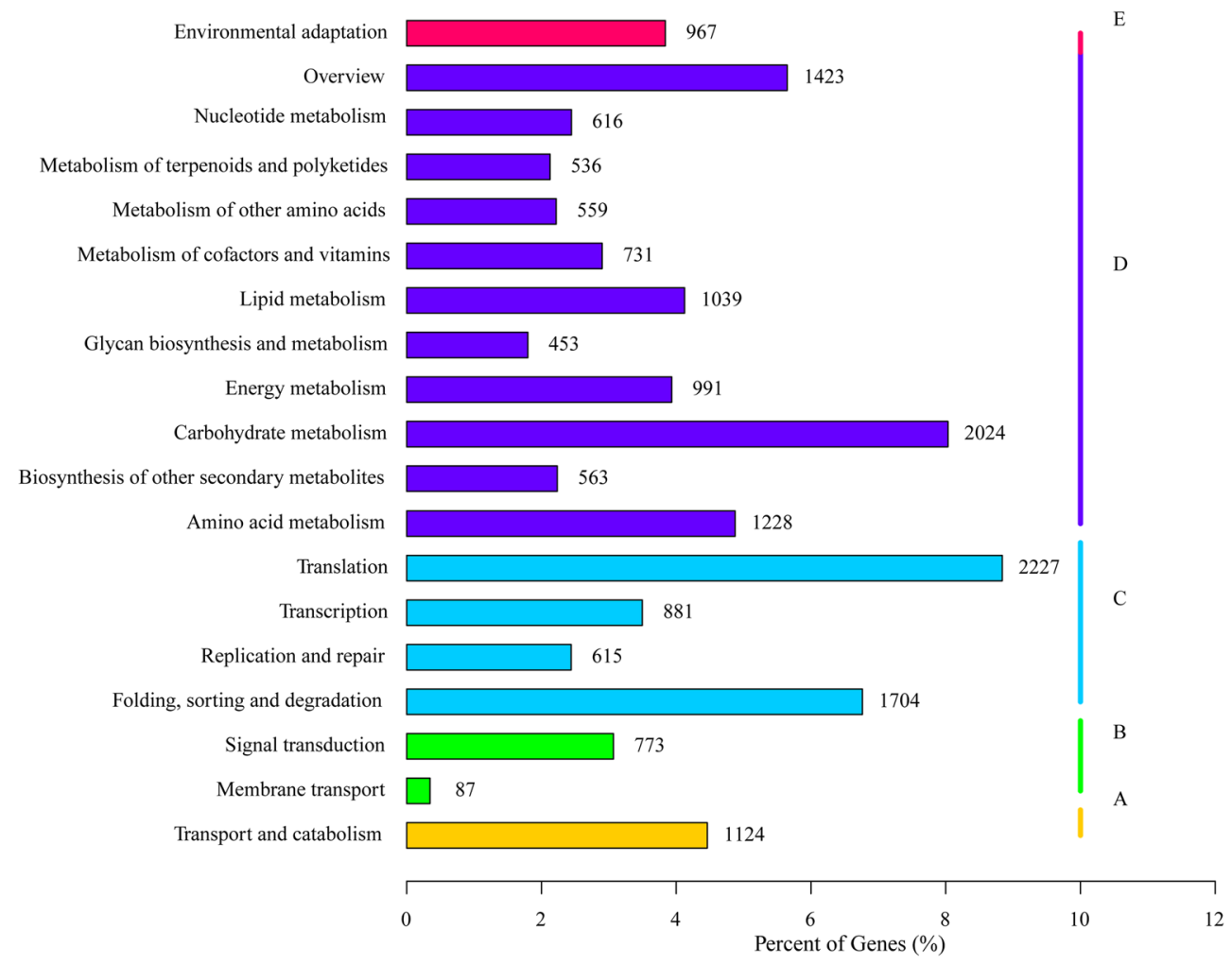

Figure 4. KEGG classifications of unigenes obtained from the C. album transcriptomic data. (A-E) Indicate the metabolic pathways cellular processes, environmental information processing, genetic information processing, metabolism, and organismal systems, respectively. The numbers represent the unigenes involved in a particular category.

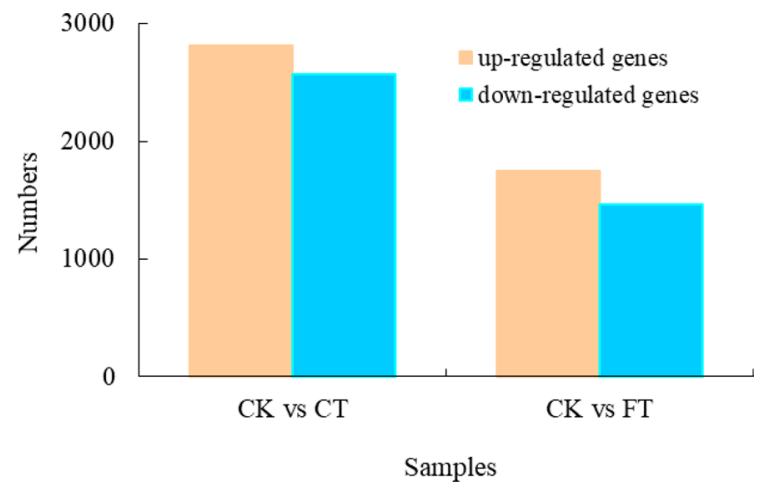

Figure 5. DEGs identified in CT and FT treatments. The graph rep presents the number of genes up and down regulated in CT and FT as compared to CK. The up-regulated genes are shown in orange, and the downregulated genes in blue.

GO enrichment analysis of DEGs. 2250 DEGs identified in CT treatment in BP category of GO, were further annotated and classified into 20 sub-categories, where DEGs involved in metabolic processes were the most abundant ones followed by the ones in single-organism process and single-organism metabolic process (Fig. 7a). 2005 DEGs identified in FT treatment were mainly classified into 20 sub-categories, mostly related to metabolic processes in general, and the metabolic processes related to single organism and organic substances. Processes related to protein phosphorylation, general metabolism, metabolism of phosphorus and phosphatecontaining compounds, oxidation-reduction reactions, single-organism metabolic processes, hormonal control, and metabolism, were all found to be enriched in CT and FT treatments (Fig. 7b). Genes involved in cell recognition, pollen-pistil interaction, recognition of pollen, pollination, cell redox homeostasis, single and multicellular organism processes, lipid metabolism, cellular homeostasis and steroid metabolism were specifically enriched in CT. Cellular proteins and other macromolecule modification processes, carbohydrate metabolism and biosyn- 


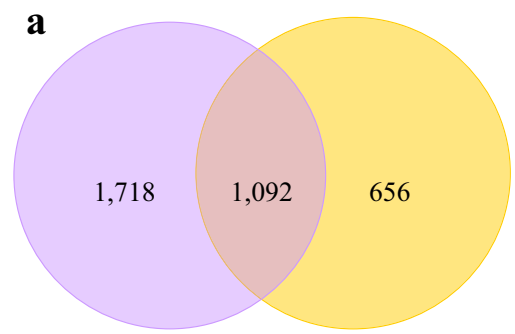

CK vs CT

CK vs FT

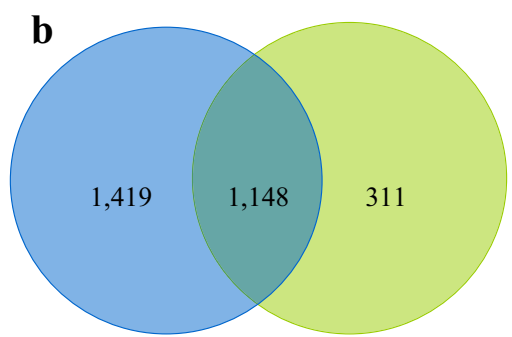

CK vs CT

Figure 6. Venn diagram for DEGs. The numbers represent the quantity of DEGs obtained in each category. (a) The number of upregulated DEGs in CT and FT compared with CK, and (b) The number of down-regulated DEGs in CT and FT compared with CK.

a
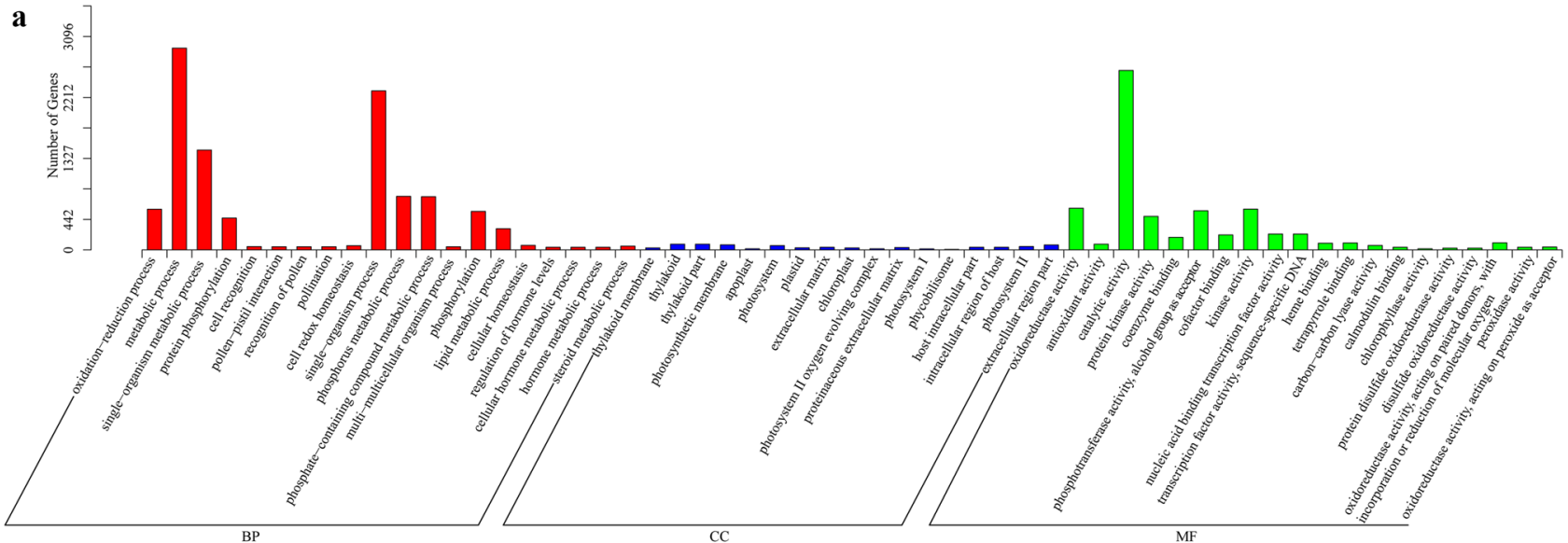

b

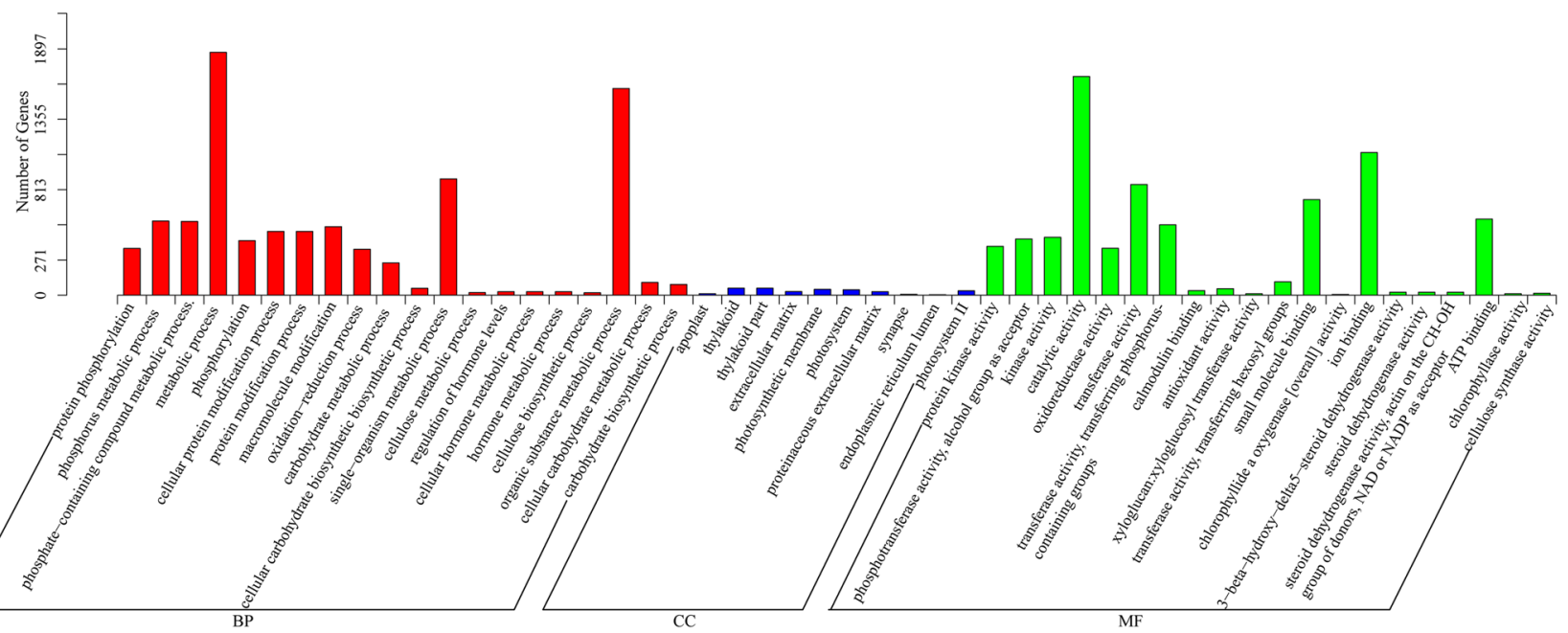

Figure 7. GO classification of DEGs under CT and FT treatments. (a) The graph shows the DEGs forming the metabolic pathways that appeared in GO analysis in CT treatment as compared to CK condition. (b) Graph showing the DEGs involved in GO classified metabolic pathways in FT treatment as compared to CK treatment. Here CK, CT and FT stand for control check, chilling stress and freezing treatments, respectively.

thesis, cellulose metabolism and biosynthesis, metabolism of organic substances, cellular carbohydrate metabolism and biosynthesis were enriched only in FT.

499 and 456 DEGs were enriched in 17 and 10 sub-categories after respective CT and FT treatments. Apoplast, thylakoid, thylakoid part, extracellular matrix, photosynthetic membrane, photosystem, proteinaceous extracellular matrix, photosystem II all were found to be enriched in both CT and FT treatments. The metabolism pathways involving plastid, chloroplast, photosystem II oxygen evolving complex, photosystem I, phycobilisome, 
a

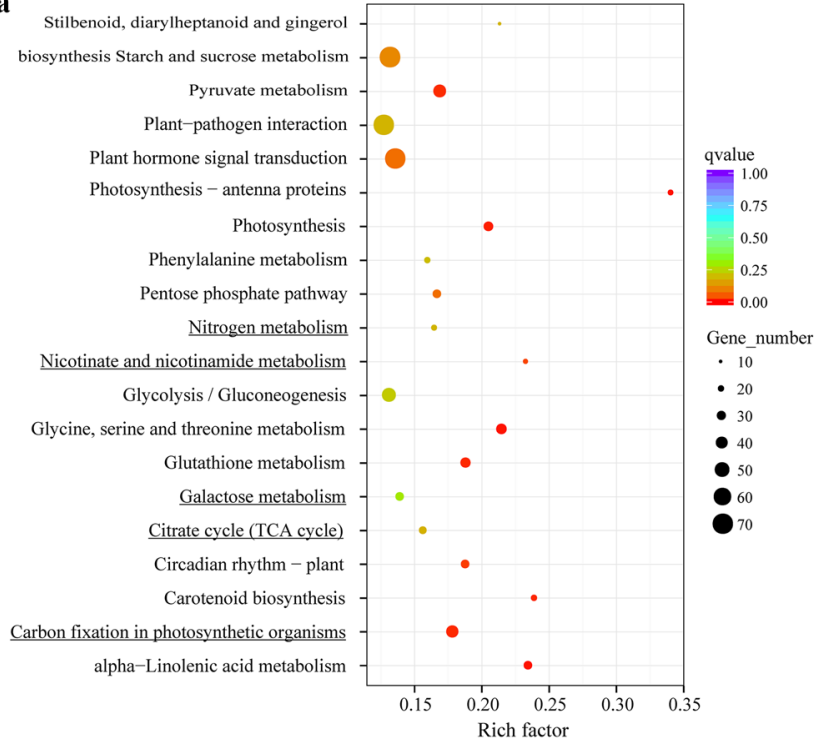

b

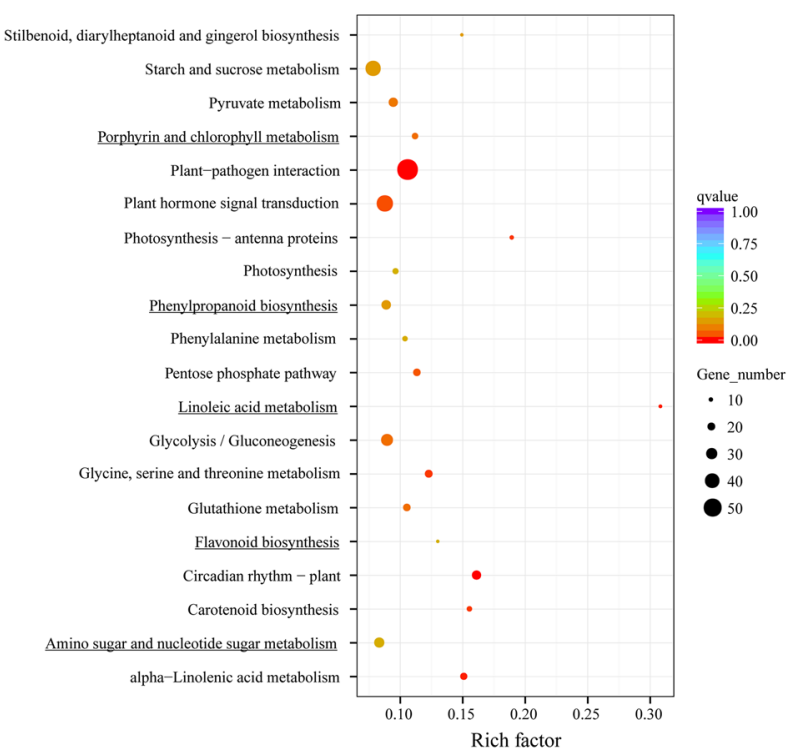

Figure 8. Twenty most enriched pathways in KEGG analysis as based on DEGs. (a) Enriched metabolic pathways after KEGG analysis of DEGs in CT treatment as compared to CK. (b) Metabolic pathways that showed up after KEGG analysis of DEGs that showed up in FT treatment with comparison to CK. The underlined pathways are specifically enriched in CT or FT when compared with CK.

host intracellular part, intracellular region of host and extracellular region were found to be strictly CT enriched, while synapse and endoplasmic reticulum lumen were only enriched in FT treatment.

A total of 1108 DEGs after CT treatment fell into 20 sub-categories in MF with the most abundant pathway related to catalytic metabolism. 965 of the observed genes that are involved in catalytic, ion binding, transferase, small molecule binding and ATP binding activities were significantly enriched in FT condition. Protein kinases, phosphotransferases with carboxyl group as acceptor, kinases, catalytic proteins, oxidoreductases, calmodulin binding proteins, antioxidant molecules, and chlorophyllase were all enriched in both, CT and FT conditions. CT specifically enriched the metabolic pathways involving various coenzymes, cofactors, nucleic acid binding transcription factors, transcription factor activity-sequence-specific DNA binding factors, heme binding factors, tetrapyrrole binding factors, carbon-carbon lyases, protein disulfide oxidoreductase, disulfide oxidoreductase and peroxidases. While only FT caused enrichment of various transferases, xyloglucan: xyloglucosyl transferase, small molecule binding factors, chlorophyllide A oxygenase [overall activity], ion binding factors, 3-beta-hydroxy-delta 5-steroid dehydrogenase, steroid dehydrogenase, ATP binding factors and cellulose synthase.

KEGG pathway analysis of DEGs. Compared with CK, the 1912 DEGs in CT treatment were found to be involved in 117 pathways whereas, the 1075 DEGs identified in FT were involved in 109 pathways. Out of the top 20 enriched pathways enriched both in CT and FT 15 pathways (Fig. 8), including photosynthesisantenna proteins, glycine/serine and threonine metabolism, alpha-linolenic acid metabolism, photosynthesis, glutathione metabolism, carotenoid biosynthesis, pyruvate metabolism, circadian rhythm-plant, plant hormone signal transduction, pentose phosphate pathway, starch and sucrose metabolism, plant-pathogen interaction, stilbenoid/diarylheptanoid and gingerol biosynthesis, phenylalanine metabolism, glycolysis /gluconeogenesis. Besides, five pathways were specifically enriched in CT, including carbon fixation in photosynthetic organisms, nicotinate and nicotinamide metabolism, citrate cycle (TCA cycle), nitrogen metabolism, and galactose metabolism. Pathways related to linoleic acid metabolism, porphyrin, and chlorophyll metabolism, phenylpropanoid biosynthesis, amino and nucleotide sugar metabolism, and flavonoid biosynthesis were found to be enriched in FT only.

Compared with CK, there were 10 metabolic pathways (involving 232 genes) significantly enriched in CT treatment $(p<0.005)$. These metabolic pathways include proteins related to photosynthesis-antenna, glycine/serine, threonine and alpha-linolenic acid metabolism, photosynthesis, carbon fixation in photosynthetic organisms, glutathione metabolism, carotenoid biosynthesis, pyruvate metabolism, circadian rhythm-plant, nicotinate and nicotinamide metabolism (Table 3). In these metabolic pathways, 18 DEGs in photosynthesis-antenna proteins were downregulated. Glycine/serine and threonine metabolism contained 17 down and 18 upregulated genes. Alpha linolenic acid metabolism enrichment showed 11 downregulated and 17 upregulated genes. Among the 32 and 41 DEGs involved in photosynthesis and carbon fixation in photosynthetic organisms, 30 and 29 DEGs, respectively, were inhibited. 22 and 12 genes were down and upregulated respectively, that are involved in glutathione metabolism whereas, 13 and 7 down and upregulated genes are involved in carotenoid biosynthesis. 18 genes were found to be inhibited and 25 gens were induced in Pyruvate metabolism. Among the 28 DEGs of circadian rhythm in plant, 25 DEGs were found to be downregulated. Nicotinate and nicotinamide metabolism contained 7 downregulated genes and 9 upregulated genes. Most of DEGs related to photosynthesis or biological rhythm management were downregulated (accounting for $85.71 \%$ of the total DEGs in these metabolic pathways) 


\begin{tabular}{|c|c|c|c|c|}
\hline Metabolism pathways & DEG number & Background number & $p$-value & DEG names \\
\hline Photosynthesis-antenna proteins & 18 & 53 & $3.076 \times 10^{-5}$ & $\begin{array}{l}\text { LHCA1, LHCA2, LHCA3, LHCA4, LHCA5, LHCB1, LHCB3, LHCB4, } \\
\text { LHCB5, LHCB6, LHCB7 }\end{array}$ \\
\hline Glycine, serine and threonine metabolism & 35 & 164 & $5.821 \times 10^{-5}$ & $\begin{array}{l}\text { AMT, AOC2, AOC3, betB, DLD, GCSH, GGAT, GLDC, GLYK, gbsA, gcvH, } \\
\text { gcvP, gcvT, glyA, gpmB, HPR1, HPR2, ilvA, ltaE, lpd, pdhD, PHGDH, PSAT1, } \\
\text { SDS, serA, serC, SHMT, tdcB, thrC, tynA, trpB, SHMT, ltaE, gcvH, GCSH }\end{array}$ \\
\hline alpha-Linolenic acid metabolism & 28 & 120 & $8.481 \times 10^{-5}$ & ACOX1, ACOX3, ACX, ADH1, AOS, DOX1, HPL, JMT, LOX2S, OPR \\
\hline Photosynthesis & 32 & 157 & $2.437 \times 10^{-4}$ & 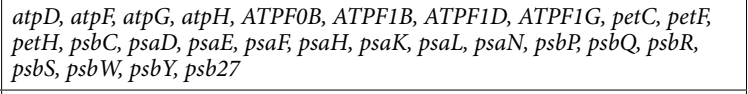 \\
\hline Carbon fixation in photosynthetic organisms & 41 & 232 & $5.082 \times 10^{-4}$ & $\begin{array}{l}\text { ALDO, FBP, GAPA, GGAT, glpX-SEBP, GOT1, GOT2, maeB, } M D H 2 \text {, } \\
N A D P^{+}, p c k A, P G K, p p c, p p d K, P R K, p r k B, r b c S, r p e, r p i A, t k t A, t k t B\end{array}$ \\
\hline Glutathione metabolism & 34 & 182 & $6.341 \times 10^{-4}$ & $\begin{array}{l}\text { APX, G6PD, GGT1/5, gnd, gntZ, gpX, gshA, gshB, GSS, GST, icd, IDH1, } \\
\text { IDH2, ODC1, pepN, PGD, RRM2, spec, speE, speF, SRM, zwf }\end{array}$ \\
\hline Carotenoid biosynthesis & 20 & 84 & $6.664 \times 10^{-4}$ & $\begin{array}{l}\text { ABA1, AOG, CCD8, crtB, crtL1, crtL2, crtP, crtQ, crtY, crtZ, YP707A, } \\
C Y P 97 C 1, \text { lcyB, lcyE, LUT1, NCED, NPQ1, PDS, VDE, ZDS, ZEP }\end{array}$ \\
\hline Pyruvate metabolism & 43 & 257 & $1.001 \times 10^{-3}$ & $\begin{array}{l}\text { ALDH, accA, aceF, ACSS, atoB, DLAT, DLD, fumB, fumC, GLO1, gloA, ldh, } \\
L D H D \text {, lpd, maeB, } M D H 2, N A D P^{+}, p c k A, P D H A, p d h C, p d h D, P K, p p c, \\
\text { ppdK, pyk }\end{array}$ \\
\hline Circadian rhythm - plant & 28 & 150 & $1.839 \times 10^{-3}$ & APRR1, CHS, CSNK2A, LHY, PHYA, PHYB, PRR5, PRR7, SPA1, TOC1 \\
\hline Nicotinate and nicotinamide metabolism & 16 & 69 & $2.775 \times 10^{-3}$ & ENPP1/3, nadA, nadB, NADK, NAPRT1, pncB, ppnK, SDT1, URH1 \\
\hline
\end{tabular}

Table 3. KEGG metabolic pathways significantly enriched in CT treatment as compared with CK.

\begin{tabular}{|c|c|c|c|c|}
\hline Metabolism pathways & DEG number & Background number & $p$-value & DEG names \\
\hline Plant-pathogen interaction & 58 & 560 & $7.501 \times 10^{-6}$ & $\begin{array}{l}\text { BAK1, CALM, CERK1, CML, CNGF, CPK, EDS1, FLS2, GK, glpK, HSP90B, } \\
\text { htpG, MEKK1P, PBS1, PR1, PTI1, RBOH, RIN4, RPS2, FLS2, MEKK1P, } \\
\text { WRKY2, WRKY33 }\end{array}$ \\
\hline Circadian rhythm - plant & 24 & 150 & $1.024 \times 10^{-5}$ & APRR1, CHS, LHY, PIF3, PRR5, PRR7, SPA1, TOC1 \\
\hline alpha-Linolenic acid metabolism & 18 & 120 & $2.583 \times 10^{-4}$ & ACOX1, ACOX3, ACX, ADH1, AOC, AOS, HPL, JMT, LOX2S, OPR \\
\hline Linoleic acid metabolism & 8 & 26 & $2.622 \times 10^{-4}$ & LOX1/5, LOX2S \\
\hline Glycine, serine and threonine metabolism & 20 & 164 & $1.308 \times 10^{-3}$ & $\begin{array}{l}\text { AGXT, AMT, AOC2, AOC3, DLD, gcvP, gcvT, GGAT, GLDC, glyA, gpmB, ilvA, } \\
\text { lpd, late, pdhD, PHGDH, SDS, sera, SHMT, tdcB, thrA, trpB, tynA }\end{array}$ \\
\hline Photosynthesis-antenna proteins & 10 & 53 & $1.326 \times 10^{-3}$ & $\begin{array}{l}\text { LHCB4 LHCA4 LHCA1 LHCA3 LHCB6 LHCA1 LHCB6 LHCB1 LHCB3 } \\
\text { LHCA2 }\end{array}$ \\
\hline Carotenoid biosynthesis & 13 & 84 & $1.383 \times 10^{-3}$ & $A B A 1, C C D 8$, crtB, crtZ, NPQ1, VDE, ZEP, CYP707A \\
\hline Plant hormone signal transduction & 45 & 524 & $2.879 \times 10^{-3}$ & $\begin{array}{l}\text { ABF, AHK2/3/4, ARF, ARR-B, BAK1, DELLA, EBF1/2, EIN3, ERF1, ERS, ETR, } \\
\text { GH3, GID1, IAA, JAR1, NPR1, PIF3, PP2C, PR1, PYL, SAUR, SNRK2, TGA, } \\
\text { K14486 }\end{array}$ \\
\hline Pentose phosphate pathway & 19 & 169 & $3.884 \times 10^{-3}$ & $\begin{array}{l}\text { ALDO, devB, FBP, G6PD, gapN, gnd, gntZ, PGD, PFK, pfkA, pgl, PGLS, PRPS, } \\
\text { prsA, rpiA, zwf }\end{array}$ \\
\hline
\end{tabular}

Table 4. Significantly enriched KEGG metabolism pathways in FT treatment as compared with CK.

whereas, other metabolic pathways regulating photosynthesis-antenna proteins, photosynthesis, carbon fixation in photosynthetic organisms, plant circadian rhythm, were found to be inhibited. The photosynthetic capacity of C. album was found to be decreased after chilling injury, and the biological rhythm adjusted to adapt to the environmental changes. Glutathione metabolism, pyruvate metabolism, nicotinate and nicotinamide metabolism are all closely related to plant antioxidant or oxidative responses. Their significant enrichment after chilling stress indicated that they play important roles in the resistive responses of C. album towards chilling stress.

Compared with CK, there were 9 significantly enriched metabolic pathways including 219 DEGs in FT treatment. These metabolic pathways include those related to plant-pathogen interaction, plant circadian rhythm, alpha-linolenic acid and linoleic acid metabolism, glycine/serine and threonine metabolism, photosynthesisantenna proteins, carotenoid biosynthesis, plant hormone signal transduction, and pentose phosphate pathway (Table 4). In these pathways, 52 of the 58 DEGs in plant-pathogen interaction were up-regulated. 20 downregulated genes and 4 up-regulated genes were involved in plant circadian rhythm. Alpha-linolenic acid metabolism pathway showed 11 down-regulated and 7 up-regulated genes, whereas 7 out of 8 DEGs in linoleic acid metabolism were up-regulated. 10 genes showed up-regulation and 10 were down-regulated in glycine/serine and threonine metabolism. 10 genes involved in photosynthesis-antenna proteins were down- regulated. 6 genes involved in carotenoid biosynthesis were down-regulated and 7 were up-regulated. Plant hormone signal transduction pathway had 23 down-regulated and 22 up-regulated genes. 11 genes were down-regulated whereas, 8 were up-regulated in pentose phosphate pathway.

In these significantly enriched metabolic pathways, those of glycine/serine and threonine metabolism, alphalinolenic acid metabolism, carotenoid biosynthesis, photosynthesis-antenna proteins, and plant circadian rhythm 
$\mathbf{a}$

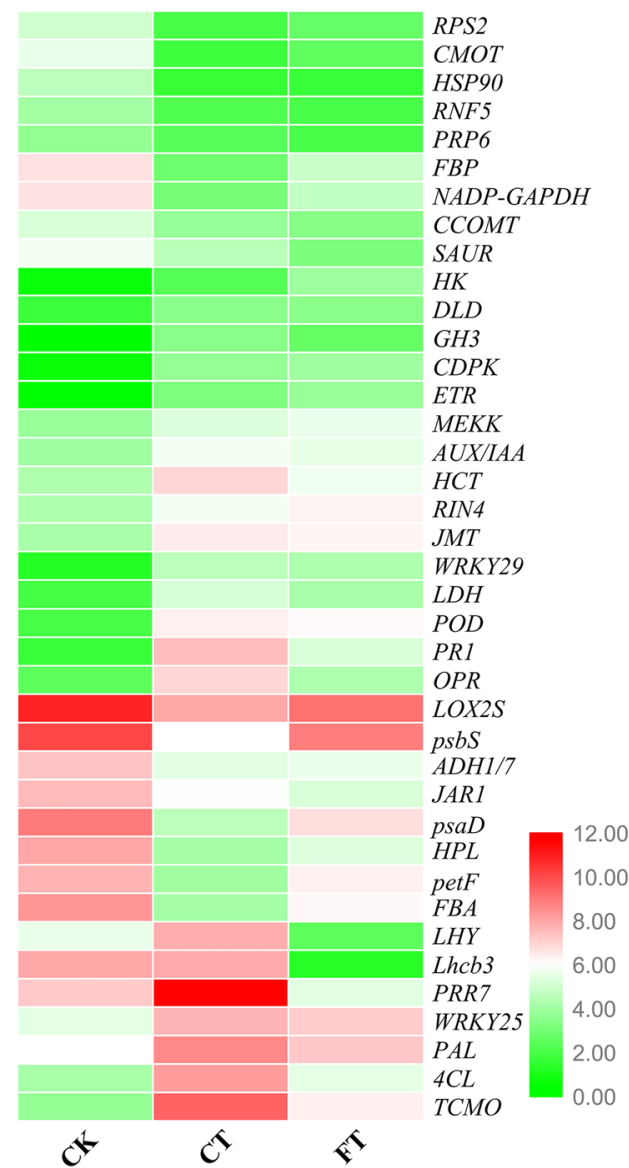

b

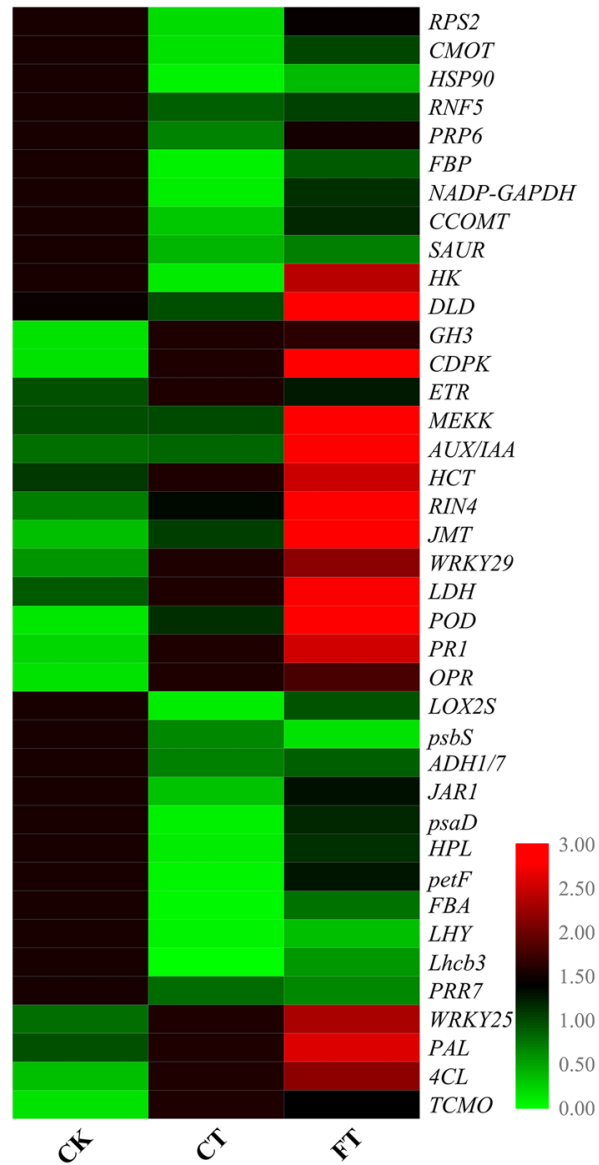

Figure 9. DEGs screening by FPKM value and qRT-PCR analysis. (a) The map shows the change in FPKM value of selected DEGs in CK, CT and FT treatments. Each column represents a given treatment and each row indicates relative expression or FPKM value of the gene written on the right side. (b) Heat map showing the relative expression of selected DEGs in CK, CT and FT conditions as measured through qRT-PCR. Gene abbreviations are being expanded in Table 5.

were significantly enriched in both CT and FT treatments. Photosynthesis, carbon fixation in photosynthetic organisms, glutathione metabolism, pyruvate metabolism, nicotinate and nicotinamide metabolism were specifically enriched in CT condition, while pathways involved in plant-pathogen interaction, linoleic acid metabolism, plant hormone signal transduction, pentose phosphate pathway were specifically enriched in FT treatment. Notably, the DEGs involved in circadian rhythms of plant and photosynthesis-antenna proteins were mostly down-regulated, indicating that both chilling and freezing temperatures inhibited these pathways in C. album. Although the number of DEGs in alpha linolenic acid metabolism, glycine/serine and threonine metabolism, carotenoid biosynthesis in chilling and freezing stress was large, the overall differences were not significant. However, compared with the chilling stress, the metabolic pathways for glutathione, pyruvate, nicotinate and nicotinamide were not significantly enriched in FT, suggesting that the changes in these metabolic pathways play more important roles in chilling responses of C. album. Alternatively, freezing treatments caused significant enrichment of genes involved in linoleic acid metabolism, pentose phosphate pathway, and plant hormone signal transduction, indicating that these pathways are involved in resistance to freezing temperature by C. album. There were especially 45 DEGs serving in pathways related to plant hormone signal transduction, suggesting that the hormones are indeed essential in developing resistance to freezing stress in C. album.

Quantitative real time PCR (qRT-PCR) analysis of DEGs. The expression levels of 39 genes, selected from enriched metabolic pathways from KEGG analysis were further studied. Compared with CK, the expression levels of 19 genes in CT and FT were significantly increased, while 20 genes were significantly downregulated both in CT and FT treatments (Fig. 9b). The relative expression levels of most of the genes verified by qRT-PCR were consistent with the RNA-Seq data (Fig. 9a) further validating the results obtained in RNA-Seq.

Verifying the DEGs involved in hormone signal transduction pathways. Under low temperature stress, plant hormone signal transduction pathway was significantly enriched in response to freezing injury but not to the chilling injury. The differential expression of 13 key genes from auxin, cytokinin, gibberellin, abscisic 

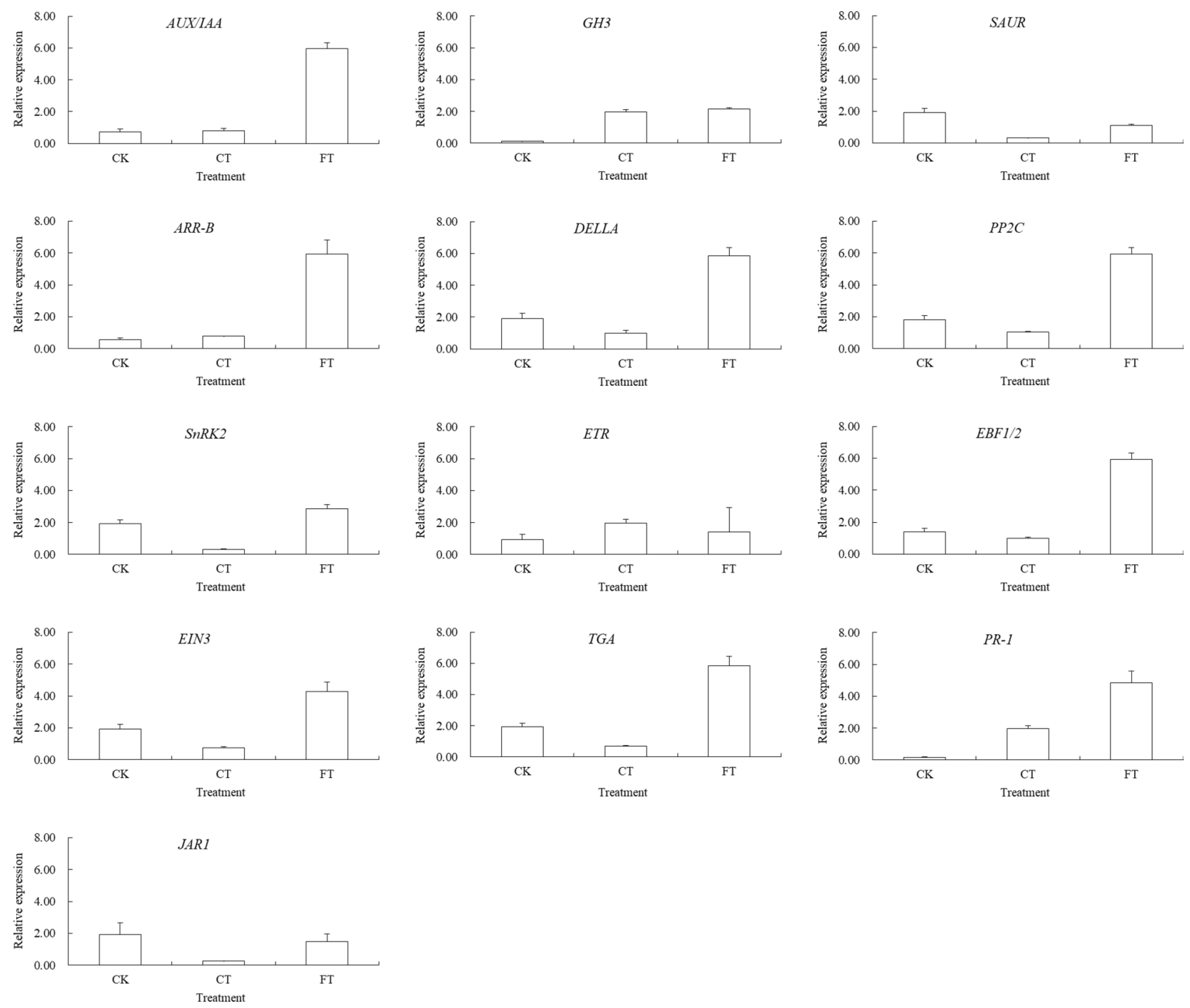

Figure 10. qRT-PCR analysis of some key genes involved plant hormone signal transduction. AUX/IAA auxin/ indole-3-acetic acid, GH3 Gretchen hagen3, SAUR small auxin-up RNA, $A R R-B$ type-B authentic response regulator, DELLA DELLA protein, PP2C $2 \mathrm{C}$ type protein phosphatases, SnRK2 sucrose non-fermenting 1-related protein kinases 2, ETR ethylene receptor, EBF1/2 EIN3 binding f-boxprotein 1/2, EIN3 ethylene insensitive 3, TGA TGA transcription factors, $P R-1$ pathogenesis related protein 1, JAR1 jasmonic acid-amino synthetase 1 .

acid, ethylene, and salicylic acid signaling pathways were also analyzed by qRT-PCR (Fig. 10). Compared with $\mathrm{CK}, \mathrm{CT}$ did not significantly change the expression level of $A u x / I A A$ in auxin signal transduction pathway. But its expression was increased more than eightfold in FT. The expression of GH3 increased 16 and 18 times respectively, in CT and FT, while the relative expression level of SAUR was decreased to about $1 / 6$ and $1 / 2$ of that in CK. Although $A R R-B$ from cytokinin signal transduction pathway increased a little in CT, the difference was not significant. However, its relative expression level in FT was increased more than 10 folds. A key gene of gibberellin signal transduction pathway, DELLA was found to be downregulated in CT, but upregulated, nearly 2 folds, in FT condition. PP2C and SnRK2, two key genes of abscisic acid signal transduction pathway, had the similar response pattern to low temperature stress, both being down-regulated in CT and up-regulated 3 and 1.5 times, respectively, in CT and FT. ETR, EBF1/2 and EIN3, three key genes of ethylene signal transduction pathway, also changed expression after exposure to low temperature. The relative expression of ETR in CT increased while that in FT did not change significantly. The expression levels of EBF1/2 and EIN3 consistently decreased in CT but increased 4 and 2 times, respectively, upon FT exposure. TGA and $P R-1$, the key genes of salicylic acid signal transduction pathway, responded to low temperature stress differently, in which TGA was significantly up-regulated ( 3 times) in CT, while $P R-1$ increased 11 and 27 times in CT and FT both, respectively. JAR1 is the key gene of jasmonate signal transduction pathway and the expression level of JAR1 also changed during low temperature stress in C. album. These results further substantiate our findings that auxin, cytokinin, gibberellin, abscisic acid, ethylene, jasmonate and salicylic acid signal transduction pathways are involved in C. album responses to low temperature stress, especially to freezing injury. 


\section{Discussion}

De novo assembly of the $C$. album transcriptome. High-throughput transcriptome sequencing, a modern biology research tool, is a common and popular method for screening DEGs, constructing metabolism networks and studying molecular regulation in plants ${ }^{20,21}$. In our study, we firstly reported the transcriptomic analysis of C. album, where a total of $18.97 \mathrm{G}$ clean data was obtained and the base error rate, Q20, Q30 and, GC content all are in accordance with the established requirements. Moreover, the $\mathrm{N}_{50}$ and $\mathrm{N}_{90}$ values for assembled and spliced genes were $2,543 \mathrm{bp}$ and $648 \mathrm{bp}$, respectively. $64.44 \%$ of unigenes were annotated in at least one of the databases- Nr, Nt, Pfam, KOG, Swiss-prot, KEGG or GO. Therefore, with this clean transcriptomic data in $C$. album we have tried to provide accurate references for future research in this area.

Comparative transcriptome analysis reveals the responses to low temperature in $C$. album. Three transcriptome libraries of C. album were constructed and the transcript changes in C. album under chilling and freezing treatments were studied. Compared with control (CK), 2810 genes were upregulated and 2567 were downregulated after chilling stress treatment (CT). Whereas 1748 and 1459 genes were up and down-regulated, respectively, after freezing stress treatment (FT). The numbers of up-regulated genes in CT and FT were both larger than that of down-regulated genes, indicating that C. album might improves cold resistance by positive regulation of these genes. Moreover, commonly up-regulated 1092 genes and 1148 down-regulated genes in both in LT and FT treatments were observed. There was a specific up-regulation and down-regulation of 1718 and 1419 genes in CT, whereas in FT the numbers were 656 and 311, respectively, indicating a mutually exclusive response in these cases. In addition, the number exclusive and non-exclusive DEGs in FT were significantly less than that in CT, which could be the reason behind the intolerance of C. album to freezing injury because of compromised life activities under freezing stress.

Pathways involved in both chilling and freezing responses in C. album. GO classification and KEGG enrichment analysis are widely used tools for transcriptomic research. The chilling and freezing responses of C. album, as seen in these analyses separately, showed some similarities but bore differences as well. The GO results showed that the shared responses mostly include DEGs related to various metabolic processes (BP category) including single-organism metabolic process, as well as different molecules catalyzing these processes. In contrast, DEGs solely related to FT, were specifically enriched in organic substance metabolic process (BP category), ion binding, transferase activity, small molecule binding and ATP binding, etc., that particularly regulate freezing acclimation in C. album. The GO classification results indicated that the responses of C. album to chilling injury and freezing injury are quite different.

Pathways that were significantly enriched after CT and FT treatments, as seen in KEGG pathway analysis were related to or involved in lysine/serine and threonine metabolism, alpha-linolenic acid metabolism, carotenoid biosynthesis, photosynthesis-antenna proteins, and plant circadian rhythm. However, those specifically enriched after CT are photosynthesis, carbon fixation in photosynthetic organisms, glutathione metabolism, pyruvate metabolism, nicotinate, and nicotinamide metabolism. FT treatment particularly enriched pathways regulating plant-pathogen interaction, linoleic acid metabolism, plant hormone signal transduction and pentose phosphate pathway. These results indicated that some of metabolic pathways were enriched in both the stresses (CT and FT) whereas some were quite exclusive to only one kind of stress exposure.

Previous reports have shown that the changes in pathways controlling plant circadian rhythm could help the plant in adjusting to a change in temperature such as cold stress ${ }^{22}$. This involves increasing expression levels of key genes involved in these pathways ${ }^{8,22,23}$. Serine and threonine protein kinases (STPK) like SpkB, SpkD, SpkE and $S P k G$, that function as transcriptional regulators have also been shown to be involved in responses towards low temperature stresses in different organisms ${ }^{24}$. Plant-pathogen interaction is closely related to cold acclimation of grape plant that is put under cold stress $\left(4^{\circ} \mathrm{C}\right)$. Such a treatment increases expression levels of CNGC, CMLs, JAZ1, Rboh, FLS2, BAK, MEKK1, MKKs, RPM1, RPS5, RIN4, PBS1, WRKYs, PR1 and MIN7 as a response to cold resistance ${ }^{25}$. The accumulation of linolenic acid in peach fruits improves its tolerance to low temperature stress ${ }^{26}$. Low temperature leads to decrease in $\mathrm{CO}_{2}$ absorption in many cold-sensitive plants, resulting in the degradation of photochemical efficiency during photosynthesis ${ }^{27,28}$. Presence of carotenoids affects the cold resistance of plants and may also be related to the changes in auxin content, oxidative damage, and membrane permeability ${ }^{29}$. Plant hormone signal transduction pathways, involving ABA, SA, JA, ETH, GA, IAA and CTK are widely recognized to play important part in cold and disease resistance, heat tolerance and other biological or abiotic stress processes in plant ${ }^{30}$. In addition, linoleic acid, pentose phosphate, glutathione, pyruvate, and nicotinamide $\mathrm{e}^{31-35}$ have all been shown to be closely related to low temperature stress responses or cold resistance in plants. All these past studies suggest that enrichment of such metabolic or signal transduction pathways are closely related to low temperature stress responses in C. album.

The plant hormone metabolism and signal transduction mediate FT tolerance in C. album. Plant hormones not only regulate the normal growth and development but are also involved in the stress responses. Many studies performed in plants like Pyrus ussuriensis, Passiflora edulis, Cymbidium goeringii, Verbena bonariensis and Fragaria ananass ${ }^{36-40}$ have shown that plants responses to low temperature are in large mediated by plant hormones.

Aux/IAA, GH3 and SAUR are the key genes for Aux/IAA-TIR1 nuclear signaling pathway. Aux/IAA dimers with Auxin Response Factor thus inhibiting its transcriptional regulation functions. GH3 encodes enzymes catalyzing auxin and amino acid coupling, and hence maintain auxin homeostasis through feedback regulation and SAUR affects functioning in some highly unstable transcriptional lines. The cold acclimation process in Arabidopsis thaliana and low temperature stress in soybean involves changes in the expression levels of auxin signal 

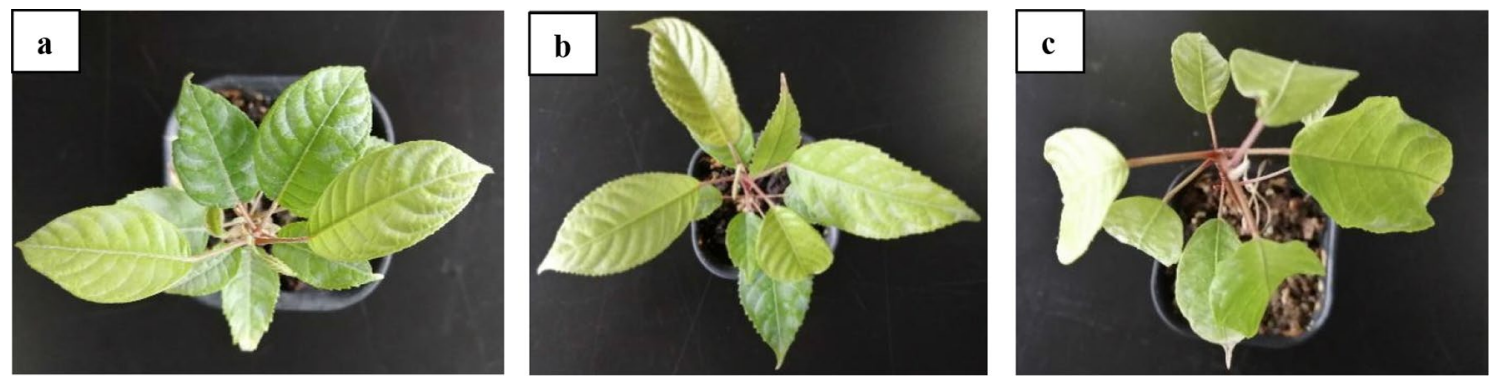

Figure 11. Seedlings of C. album treated at different temperatures for $24 \mathrm{~h}$. (a-c)show the seedlings of C. album that were given treatments for control check $\left(25^{\circ} \mathrm{C}\right)$, chilling stress, $\mathrm{CT}\left(4^{\circ} \mathrm{C}\right)$ and freezing stress, FT $\left(-3{ }^{\circ} \mathrm{C}\right)$ for $24 \mathrm{~h}$, respectively.

transduction related gene $A u x / I A A^{42,43}$. In addition, overexpression of $G H 3$ in rice increases its cold resistance ${ }^{44}$ and many PtSAUR genes in Populus tomentosa are downregulated after low temperature stress ${ }^{45}$. Moreover, in rice plant the different members of these enzymatic families mediate different responses to low temperature stress ${ }^{41}$. For example, under low temperatures IAA9, IAA20, GH3-1, GH3-8, and SAUR21 are upregulated whereas, IAA10, IAA25, and SAUR57 are downregulated. The expression patterns of IAA9, IAA20, GH3-1, GH3-8 and SAUR57 in rice are identical to those induced in C. album under low temperature stress. Our results are consistent with these old findings further supporting the claim that $A u x / I A A, G H 3$ and SAUR are indeed involved in low temperature stress responses of C. album.

Cytokinin is an important plant hormone that regulates plant growth and development as well as responses to abiotic stress though key genes like $A R R-A$ and $A R R-B$. The expression of $A R R 18$ gene, a positive regulator of low temperature responses, is significantly increased in rubber plant after low temperature treatment ${ }^{5}$. We also found an increased expression level of $A R R-B$ gene in C. album after chilling and freezing stress, which positively correlated with the extent of temperature downregulation.

A protein named DELLA is the core component of gibberellin signal transduction pathway and plays a negative role in regulating GA signal transduction pathway ${ }^{48}$. Gibberellin plays an important role in plant responses to low temperature stress ${ }^{46,47}$. DELLA enables plant survival through adverse environmental conditions by regulating plant hormones ${ }^{49}$. The tolerance of plants to stress depends on the amount of DELLA in vivo. When the active gibberellin is limiting, DELLA level increases and the tolerance to stress improves. In tomato, the crosstalk of PIF4 (Phytochrome interaction 4) and DELLA regulates CBF transcription and hormone homeostasis during cold stress response by integrating light and temperature signaling and sharing of hormone pathways and transcription regulators thus enabling better adaptation of plants to cold stress ${ }^{50}$. We also found an increased expression of DELLA in qRT-PCR after freezing injury, suggesting its role in negatively regulating gibberellin content in vivo in C. album to adapt to low temperature stress.

SnRK2 is a plant specific protein kinase, which plays an important role in ABA signal transduction and osmotic stress regulation $^{51}$. Low temperature could induce the $S n R K 2$ gene in tobacco and wheat ${ }^{52,53}$ and overexpression of TaSnRK2.4 in Arabidopsis improves its cold resistance through a series of physiological changes such as reduction in rate of water loss, enhanced stability of cell membrane and improvement in the photosynthetic and osmotic potential ${ }^{54} . P P 2 C$ is a serine/threonine residue protein phosphatase and a core negative regulator of ABA signal transduction. In Arabidopsis, $P P 2 C$ has also been shown to be closely related to the low temperature acclimation $^{55}$. An enrichment of ABA signaling pathway in our study further confirms its involvement in low temperature stress responses and subsequently validates our results too.

Being the key enzyme for jasmonate signal transduction pathway, JAR1 catalyzes the last step forming molecularly active JA-Ile from the combination of jasmonate and amino acids, especially isoleucine ${ }^{56}$. LOX, AOS and JMT are other key enzymes in jasmonate biosynthesis or signal transduction pathway. Previous studies have shown that low temperature could induce the expression of LOX, AOC and JAR1 genes in Artemisia annua, thus increasing the level of endogenous jasmonate, which consequently induces the expression of ETR1, ETR2 and $A P 2 / E R F^{57}$. We also found an increased expression of these genes in C. album, as response to chilling or freezing injury.

$E T R, E B F 1 / 2$ and EIN3 are genes serving in ethylene signal transduction pathway. After cold stress in kiwifruit and papaya, expression levels of some of the ETR and EIN3 members are increased, with a slight change in the expression level of $E B F$ as well ${ }^{58,59}$. As a key member of salicylic acid signal transduction pathway, transcription factor TGA is involved in temperature stress responses in some plants ${ }^{60}$, while $P R-1$ gets expressed in others ${ }^{61}$. Our results corroborate with these earlier findings further strengthening the role of ethylene and salicylic acid signal transduction pathways in cold stress responses in C. album.

\section{Materials and methods}

Plant materials and low temperature treatments. Healthy, strong and uniform C. album. cv. 'Fulan 1 ' seedlings of height of $\sim 20 \mathrm{~cm}$ were selected and kept in growth chambers (GXZ-280C, Ningbo, China) maintained at $25^{\circ} \mathrm{C}, 60 \%$ to $80 \%$ relative humidity, and a photoperiod of $12 \mathrm{~h}(2000 \pm 200 \mathrm{~lx})$ for one week. Then the seedlings were subjected to three different temperature treatments, i.e. freezing stress treatment $\left(\mathrm{FT},-3^{\circ} \mathrm{C}\right)$ in freezer, chilling stress treatment $\left(\mathrm{CT}, 4^{\circ} \mathrm{C}\right)$ in freezer and control check treatment $\left(\mathrm{CK}, 25^{\circ} \mathrm{C}\right)$ in growth chambers for $24 \mathrm{~h}$. Ten seedlings were collected for each treatment and each experiment was repeated 3 times. During 
the whole treatment process, all seedlings were grown in the same conditions as formal, and the samples were simultaneously collected in dark after treatment. Compared with the seedlings in CK (Fig. 11a), the leaves from seedlings in FT experienced dehydration and gradually wilted (Fig. 11c), while there was no obvious phenotypic change in leaves from seedlings grown in CT (Fig. 11b). After the $24 \mathrm{~h}$ temperature treatment of the seedlings, the $3 \mathrm{rd}$ and 4 th leaves from top to base were collected (from all the replicative experiments) and instantly frozen in liquid nitrogen. The samples were stored in the freezer at $-80^{\circ} \mathrm{C}$ till further use. The collection of plant material in this research, complied with relevant institutional, national, and international guidelines and legislation.

Total RNA extraction and detection. Total RNA was extracted by E.Z.N.A. Plant RNA Kit (OMEGA bio-tek, USA) as per the manufacturer's instructions. The RNA was checked for quality and quantity by first running it on a $1.5 \%$ agarose gel, followed by a test for purity, concentration and integrity using the TU-1810 spectrophotometer (Puxi, CHN), NanoDrop OneC Flurometer (Thermo Fisher Scientific, USA) and the Agilent Bioanalyzer 2100 system (Agilent Technologies, USA). Only high-quality total RNA was used for further experiments including the library construction.

Library preparation for RNA-Seq. $\quad 1.5 \mu \mathrm{g}$ of high-quality total RNA, treated at different temperatures was used for library preparation and NEBNext Ultra RNA Library Prep Kit for Illumina (NEB, USA) was used to generate the sequencing libraries and the library preparation and RNA-Seq were carried out by Novogene, Beijing, China ${ }^{62}$. Briefly, mRNA was made from the total RNA using poly-T oligo-attached magnetic beads followed by first strand cDNA synthesis using random hexamer primer and M-MuLV Reverse Transcriptase (RNase H-) as per the manufacturer's instructions. DNA polymerase I and RNase $\mathrm{H}$ were subsequently added for the synthesis of second strand cDNA and the removal of mRNA template. Remaining overhangs were converted into blunt ends by exonuclease/polymerase reactions. After adenylation of 3' ends of the generated cDNA fragments, NEBNext Adaptor, with hairpin loop structure, were prepared for hybridization. AMPure XP system (Beckman Coulter, USA) was used to purify library fragments where all 150-200 bp cDNA fragments were selected. Then, $3 \mu \mathrm{L}$ of size selected USER Enzyme (NEB, USA) was used for adaptor-ligation of cDNA at $37^{\circ} \mathrm{C}$ for 15 min followed by $5 \mathrm{~min}$ of heating at $95^{\circ} \mathrm{C}$ before PCR analysis. PCR was performed with Phusion High-Fidelity DNA polymerase, Universal PCR primers and Index (X) Primer according to the mentioned instructions from the manufacturer. Lastly, PCR products were purified by AMPure XP system and library quality was evaluated on Agilent Bioanalyzer 2100 system.

Clustering, sequencing, and data analysis. Clustering of the indexed-coded samples was done via cBot Cluster Generation System that uses TruSeq PE Cluster Kit v3-cBot-HS from Illumina. The library preparations were sequenced on Illumina Hiseq-2000 platform and paired-end reads were generated after the cluster generation. Followed processing of fastq formatted raw data (raw reads) by internal perl scripts, and clean data (clean reads) were obtained by removing reads with adapter, ploy- $\mathrm{N}$ and low-quality raw data. Subsequently, $\mathrm{Q}_{20}, \mathrm{Q}_{30}$, GC-content and sequence duplication level for the clean data were calculated. The high-quality clean data were then used for downstream analysis. Transcriptome was assembled using Trinity with min_kmer_cov default value of 2 and all other parameters were set to their respective default values ${ }^{63}$.

Gene function was annotated using Nr, Nt, Pfam, KOG, Swiss-Prot, KEGG and GO databases. The read counts for each sequenced library was adjusted by edgeR program package through one scaling normalized factor. Differential expression analysis of two samples was performed using the DEGseq R package as shown previously ${ }^{64}$. $\mathrm{P}$ value was adjusted by using the $\mathrm{q}$ value ${ }^{65} . q$-value $<0.005$ and $\left|\log _{2}{ }^{\text {(fold change) }}\right|>1$ was set as the threshold for calculating significantly differential expression of a gene.

GO enrichment analysis of the differentially expressed genes (DEGs) was performed according to the GOseq $\mathrm{R}$ packages based Wallenius non-central hyper-geometric distribution ${ }^{22}$. The statistical enrichment of DEGs in KEGG pathways was tested by KOBAS software as shown previously ${ }^{66,67}$.

qRT-PCR analysis of DEGs. TransScript All-in-One First-Strand cDNA Synthesis SuperMix kit (TransGen Biotech, CHN) was used to synthsize high quality cDNA for qRT-PCR analysis. cDNA samples of CK, CT and FT were mixed equally, and then gradiently diluted 4 times to obtain a standard curve (dilutions: $10^{-1}, 40^{-1}, 160^{-1}$ and $640^{-1}$ ) using a suitable annealing temperature, that was screened according to the amplification efficiency. Eppendorf Realplex 4 and TransStart Top Green qPCR SuperMix were used to detect the relative expression of the selected 39 genes (including 13 plant hormone signaling and transduction pathway related genes, i.e. AUX/ $I A A, G H 3$ and $S A U R$ involving in auxin signaling pathway, $A R R-B$ involving in cytokinin signaling pathway, $D E L L A$ involving in gibberellin signaling pathway, $P P 2 C$ and $S n R K 2$ involving in abscisic acid signaling pathway, ETR, EBF1/2 and EIN3 involving in ethylene signaling pathway, TGA and $P R-1$ involving in salicylic acid signaling pathway and JAR1 involving in jasmonate signaling pathway) in different treatments by using primers as listed in Table 5. The amplification cycle of qRT-PCR: pre-denaturation at $94^{\circ} \mathrm{C}$ for $30 \mathrm{~s}$; denaturation at $94^{\circ} \mathrm{C}$ for $10 \mathrm{~s}$, annealing at $58-60^{\circ} \mathrm{C}$ for $15 \mathrm{~s}$, extension at $72^{\circ} \mathrm{C}$ for $10 \mathrm{~s}, 40$ cycles. The sample was maintained $94^{\circ} \mathrm{C}$ for $15 \mathrm{~s}$, at $60^{\circ} \mathrm{C}$ for $15 \mathrm{~s}$, and then the temperature was raised to $94^{\circ} \mathrm{C}$ for $15 \mathrm{~s}$ at the rate of $0.11^{\circ} \mathrm{C} / \mathrm{s}$ to draw the 


\begin{tabular}{|c|c|c|c|}
\hline Gene ID & Gene name & Primer sequence (5'-3') & Description \\
\hline \multirow{2}{*}{ Cluster-7340.51676 } & \multirow{2}{*}{ PRR7 } & ATCACAACAGCAGCCTCCT & \multirow{2}{*}{ Pseudo-response regulator 7} \\
\hline & & TTCCTGAGCAAAGCACTGAC & \\
\hline \multirow{2}{*}{ Cluster-7340.66287 } & \multirow{2}{*}{$L H Y$} & AAATCTTCCCACCTCCGAG & \multirow{2}{*}{ Late elongated hypocoty } \\
\hline & & CСCTCTTCAGAAACTTCCTTCC & \\
\hline \multirow{2}{*}{ Cluster-7340.85283 } & \multirow{2}{*}{ Lhcb3 } & ACCCTCTTAGAAATGTTGCCTC & \multirow{2}{*}{ Light-harvesting chlorophyll a/B-protein } \\
\hline & & TGTCCCATCCATAGTCACCTG & \\
\hline \multirow{2}{*}{ Cluster-7340.42050 } & \multirow{2}{*}{$D L D$} & TGTTGTTCTTGTCTCCGCTG & \multirow{2}{*}{ Dihydrolipoamide dehydrogenase } \\
\hline & & CCATCCTCTTCTGCTTTGTG & \\
\hline \multirow{2}{*}{ Cluster-7340.48264 } & \multirow{2}{*}{$C D P K$} & CAACGGAGACTTGACATTTGAC & \multirow{2}{*}{ Calcium-dependent protein kinase } \\
\hline & & GCTTCCAGTAACATTCGCAC & \\
\hline \multirow{2}{*}{ Cluster-7340.41917 } & \multirow{2}{*}{ MEKK } & GGAATGTATGCCAGCGTTG & \multirow{2}{*}{ Mitogen-activated protein kinase kinase kinase } \\
\hline & & CCTCTTCACAAATGGATGGTC & \\
\hline \multirow{2}{*}{ Cluster-7340.50488 } & WRKY 25 & CСАТСТСТССТССТССТGTT & WRKY transcrintion factor 25 \\
\hline & $W R K Y Z 3$ & GGTTATCCCAGTTGAAGGTTTG & WKK T transcription ractor 20 \\
\hline & & CAGATGTAGCAGTTCAAAGGGT & WRKY transcrintion factor 29 \\
\hline Cluster-7340.38428 & WRKY 29 & AGTGAGTTTCGGTGGGTAGG & WRKY transcription factor 29 \\
\hline & & ATGGGAAGGGAAGGGTTT & \\
\hline Cluster-7340.58469 & RIN4 & CATCAGGACTTTCATCGCC & RPM1-interacting protein 4 \\
\hline Cluster- 7340.37043 & $H S P 90$ & CGATGATGAAGATGATGAGCC & Heat shock protein 90 \\
\hline (1uster-1540.3/043 & ПОРY0 & СCTCCTTGGTGATTTCCTCTG & 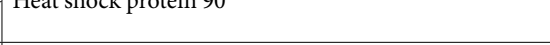 \\
\hline Cluster-7340 74583 & $R P S 2$ & GAAATCTTATGCCAACCCTACC & Resistance to P syringae 2 \\
\hline Cluster-1540./4583 & KPSZ & TCCGTCATCACATCCGTTT & Kesistance to P. syrmgae 2 \\
\hline Cluster-7340 51547 & $I O X 2 S$ & CAAGTTCAGCCAAGACCCTCT & Iinoxyoenase \\
\hline Cluster-1540.5154/ & LUNZS & CAACAGCCGTAACCTTCAAAG & Lipoxygenase \\
\hline Cluster -734055174 & $H P I$ & GAAACTCTCCGACTCAACCC & Hydroneroxide lyase \\
\hline Cluster-1340.551/4 & HPL & ATACССACAAAGCAACTCTCC & Hydroperoxide lyase \\
\hline Cluster-7340 50441 & $A O S$ & GGTTTGTTGGTGGAGAAGGT & 9-allene oxide synthase \\
\hline C tuster-1040.50441 & $A O S$ & CACTGTTTATTCCCTGCGG & y-allene oxrce syntnase \\
\hline Cluster-7340.57514 & $O P R$ & GGCTATCTCCCTTTGCTGAC & 12-oxo-phytodienoic acid reductase \\
\hline Cluster-1540.5/514 & OTK & TTTCATTCTGGGCTCAACC & 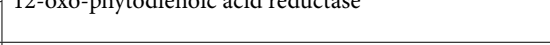 \\
\hline & & CGGTTCTTTCTATGGCAGG & Jasmonate O-methyltransferase \\
\hline Cluster-7340.40449 & $J M T$ & TGTGCTGGCGATGTAAATG & Jasmonate U-metnytransierase \\
\hline Cluster-7340 58766 & psbs & TCTCTCAACTTCTGCTCAGTCC & Photosystem I subunit IJ \\
\hline Cluster-1040.50/00 & $p s 00$ & CCATCTTCAACCTTAGGCTTAG & 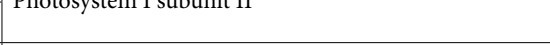 \\
\hline Cluster-7340 38192 & $p s a D$ & AGTCCCAAACATCTCACCTTC & Photosystem I subunit IJ \\
\hline Cluster-1340.38192 & psad & TCCAGTGCTTCCTCCAAAG & Pnotosystem I suounit il \\
\hline Cluster-7340,46508 & petF & GCCGTTACAAGCCTCAAGTC & Protein-protein interaction of GcpE protein with ferre- \\
\hline & petr & CTCAGCGTGGTCAAGAATGT & doxin I \\
\hline Cluster-7340 50425 & $H C T$ & TCCCAGCGTCTACTTCTATCG & Shikimate n-hydroxycinnamovl transferase \\
\hline (1uster-1540.50425 & $\Pi \mathbb{C}_{1}$ & ATСССТСТTСААССТTCСG & 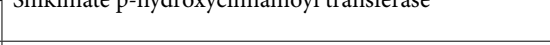 \\
\hline Cluster-7340 49158 & & AACCACTACCAGCACTTGATTC & Caffeovl-Co A O-metbyltransferase \\
\hline Cluster-7340.49158 & CCOMT & \begin{tabular}{|l|} 
GCAGTAACATCCCTCATCTCC \\
\end{tabular} & Cafteoyl-CoA O-methyltransterase \\
\hline Cluster-7340 49770 & $P O D$ & GAGTTGCTGCCTTCATTCTT & Peroxidase \\
\hline 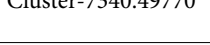 & $P O D$ & GCTGCTTCCACCTGAGTTT & \\
\hline Cluster-7340 3616 & CMOT & TGTATCGCCAACTGAAATCG & 2-(4-chloro-3-methylnhenvl)-2-oxoettyl thiocyanate \\
\hline Cluster-/340.3616 & CMOT & GGCTACGCAAAGAGCAAGT & 2-(4-cnioro-3-metnylpnenyi)-2-oxoetnyi tniocyanate \\
\hline Cluster-7340 51517 & $4 C I$ & AAACAAGGGTGGCTACATACG & 4-coumarate_CoA ligase \\
\hline Cluster-7340.51517 & $4 C L$ & CATCTTTCATTGGGACTACTGC & 4-coumarate-CoA ligase \\
\hline Cluster-7340 50851 & $T C M O$ & ACATCCTCCTTCTCCGCAT & Trans-cinnamate 4-monooxyonase \\
\hline 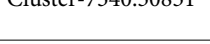 & THNO & ССТССТСАТСТТССТССААТ & 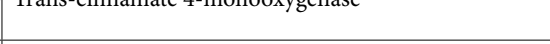 \\
\hline Cluster-7340 50096 & $P A I$ & CCCAAGAAGCCTCCAAACT & Phenvlalanine ammonia-lyase \\
\hline C & PAL & AACACAGCCAGAACATTAGCC & Pnenylalanine ammonia-ryase \\
\hline Cluster-734066106 & $H K$ & TGCATGAGGATGACACTCC & Hexokinase \\
\hline Cluster-1340.66106 & HK & CCAAGGAAGGAGACAAGCA & Hexokinase \\
\hline Cluster-7340.18603 & $F B P$ & AAGTTCGTCTGCTCTGCTGTT & Fructose-1,6-bisphosphatase \\
\hline Cluster-1040.18003 & $F D P$ & GTTGCCTCTTCATCTTCTTCAG & Fructose-1,0-0ispnospnatase \\
\hline
\end{tabular}




\begin{tabular}{|c|c|c|c|}
\hline Gene ID & Gene name & Primer sequence $\left(5^{\prime}-3^{\prime}\right)$ & Description \\
\hline \multirow{2}{*}{ Cluster-7340.50923 } & \multirow{2}{*}{$F B A$} & CCCATCTGCCCTTACTGTTC & \multirow{2}{*}{ Fructose-bisphosphate aldolase } \\
\hline & & \begin{tabular}{|l|} 
GGTTAGCCTCGGTGTTCTCT \\
\end{tabular} & \\
\hline \multirow{2}{*}{ Cluster-7340.66723 } & \multirow{2}{*}{ NADP-GAPDH } & TTTAGCCATCCCACCGTT & \multirow{2}{*}{ NADP-glyceraldehyde-3-phosphate dehydrogenase } \\
\hline & & GAGCCTTTCCCAGTGATACAG & \\
\hline \multirow{2}{*}{ Cluster-7340.43174 } & \multirow{2}{*}{$L D H$} & AATCCACCCAGTTTCAGTCC & \multirow{2}{*}{ Lactate dehydrogenase } \\
\hline & & \begin{tabular}{|l|} 
CGTTTAGCCTCCTCGTCACT \\
\end{tabular} & \\
\hline \multirow{2}{*}{ Cluster-7340.37158 } & \multirow{2}{*}{$A D H 1 / 7$} & ССТСТАСАСАТСССТTTGCC & \multirow{2}{*}{ Alcohol dehydrogenase } \\
\hline & & AACGCTCTCCACAATCCCT & \\
\hline \multirow{2}{*}{ Cluster-7340.33488 } & \multirow{2}{*}{$A U X / I A A$} & AGGAGGACGAGGATGTTACTTT & \multirow{2}{*}{ Auxin/indole-3-acetic acid } \\
\hline & & CTGTCAACGGAAGAGCAGTC & \\
\hline \multirow{2}{*}{ Cluster-7340.64060 } & \multirow{2}{*}{ GH3 } & GCTGTTGATTCTGCCCTTTC & \multirow{2}{*}{ Gretchen hagen 3} \\
\hline & & GCGTTTCGGCTTAGGATTT & \\
\hline \multirow{2}{*}{ Cluster-7340.51543 } & \multirow{2}{*}{ SAUR } & TGCTCTGCTAACTCCAAATCC & \multirow{2}{*}{ Small auxin-up RNA } \\
\hline & & ACTCCCTTCTGCTCGTATCC & \\
\hline \multirow{2}{*}{ Cluster-7340.49359 } & \multirow{2}{*}{ ETR } & TTGACCCTATCTTCTCGGTTG & \multirow{2}{*}{ Ethylene receptor } \\
\hline & & CAATCCAAATGTGACCTCCC & \\
\hline \multirow{2}{*}{ Cluster-7340.53982 } & \multirow{2}{*}{ JAR1 } & AATGTATTCCGCAGTTCCC & \multirow{2}{*}{ Jasmonic acid-amino synthetase 1} \\
\hline & & TAAGCCCACAGAGGAGATGG & \\
\hline \multirow{2}{*}{ Cluster-7340.26872 } & DNIE & ATCCCTCCCGTTGTCTTCT & F3 ubiouitin-nrotein ligase \\
\hline & $R N F 5$ & GCGACCTGCTTGTTGATTC & E3 ubiquitin-protein ligase \\
\hline Clucter 734044794 & $P R D G$ & CCGTTCTGCCACCACTATT & Pre mPNA nocescing foctor 6 \\
\hline Cluster-7340.44794 & PRP6 & \begin{tabular}{|l|} 
CGGCTTCTTTATCATCCTCGT \\
\end{tabular} & Pre-mRNA-processing factor 6 \\
\hline & & TCAACAAAGTGCTGGCGT & Tyne-B uuthentic recnonce regulator \\
\hline Cluster-7340.72327 & $A R R-B$ & CATTCTCCTGTCCATCCTCTT & Type-B authentic response regulator \\
\hline Clucter 724050240 & DEI 4 & TGAGCAAGAGACGAACCATAAC & DFU A notain \\
\hline Cluster-7340.59249 & DELLA & \begin{tabular}{|l} 
GCTTCCCTAAATACACCTCGG \\
\end{tabular} & DELLA protein \\
\hline & & TTGTTCTCCCTCGGTTCAT & SC twpe nrotein nhesnhatases \\
\hline Cluster-7340.60183 & $P P 2 C$ & \begin{tabular}{|l} 
TCCCATTCAAACTCACTCTGAC \\
\end{tabular} & 2C type protein phosphatases \\
\hline Clucter 723020010 & $\operatorname{cs} D V 2$ & GGCAACAATAATGGACCGT & Sucrom \\
\hline Cluster-7340.20010 & SnRK2 & \begin{tabular}{|l} 
TGGTTCATAATCTCCCTCTGC \\
\end{tabular} & Sucrose non-fermenting 1-related protein kinases 2 \\
\hline Clucter 734050447 & $T C A$ & GGTCAATCTACTGCTATCGTGG & 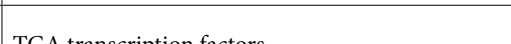 \\
\hline Cluster-7340.50447 & $T G A$ & \begin{tabular}{|l|} 
CTTTCTTCCTCAATCGGCTT \\
\end{tabular} & TGA transcription factors \\
\hline$C l$ locton 721000102 & $\angle P F 1 / 2$ & CATAGCCTGTGGATGTCCGT & CIN2 binding 5 bov notain \\
\hline Cluster-7340.60183 & EBFI/2 & AAGGGCATTGGCAAAGTTC & EIN3-binding F-box protein \\
\hline Clucter 724050717 & $F I N Z^{2}$ & GAAGAAGATGTCCCGTGCTC & Fthulone incomcitive nrotein 3 \\
\hline Cluster- 7340.50747 & EIN3 & ATTATCAGATGCTCCACTCACC & Ethylene-insensitive protein 3 \\
\hline$C l=0$ & DD1 & GGCACTACACTCAGGTGGTTT & \\
\hline Cluster-7340.43403 & PR1 & \begin{tabular}{|l} 
GCATCCAATGAAAGTTCCTCC \\
\end{tabular} & Pathogenesis-related protein 1 \\
\hline
\end{tabular}

Table 5. List of qRT-PCR primers used in the study.

dissolution curve. The reaction was set as with $10 \mu \mathrm{L} 2 \times$ TransStart Top Green qPCR SuperMix, $0.4 \mu \mathrm{L}$ Passive Reference Dye $(50 \times), 0.2 \mu \mathrm{M}$ each upstream and downstream primer, and $0.5 \mu \mathrm{M}$ cDNA template. ACTB7 and TUB5 were used as reference genes ${ }^{68}$. SPSS19.0 was used for analyzing the results statistically. TBtools was used to draw heatmaps according to the relative expression and FPKM value of the genes.

Received: 14 July 2020; Accepted: 5 May 2021

Published online: 18 May 2021

\section{References}

1. Ding, Y., Shi, Y. \& Yang, S. Advances and challenges in uncovering cold tolerance regulatory mechanisms in plants. New Phytol. 222, 1690-1704 (2019).

2. Ding, Y., Shi, Y. \& Yang, S. Molecular regulation of plant responses to environmental temperatures. Mol. Plant 13, 544-564 (2020).

3. Zhang, C. et al. Functional conservation analysis and expression modes of grape anthocyanin synthesis genes responsive to low temperature stress. Gene 574, 168-177 (2015).

4. Li, X. L. et al. Genome-wide analysis of antioxidant enzyme gene families involved in drought and low-temperature responses in apple (Malus domestica). J. Hortic. Sci. Biotechnol. 93, 337-346 (2018).

5. Gong, X. X. et al. Transcriptome profiling of rubber tree (Hevea brasiliensis) discovers candidate regulators of the cold stress response. Genes Genomics 40, 1181-1197 (2018). 
6. Klepikova, A. V. \& Penin, A. A. Gene expression maps in plants: Current state and prospects. Plants 8, 309 (2018).

7. Liu J, Xu Y, Zhang L, Li W, Cai Z, Li F, Peng M, Li F, Hu B. De novo assembly and analysis of the transcriptome of Rumex patientia L. during cold stress. PLoS ONE, 12, e0186470 (2017).

8. Li, J. et al. De novo transcriptome sequencing and the hypothetical cold response mode of Saussurea involucrata in extreme cold environments. Int. J. Mol. Sci. 18, 1155 (2017).

9. Maia, L. C. D. et al. Transcriptome profiling of rice seedlings under cold stress. Funct. Plant Biol. 44, 419 (2017).

10. Meng, D. et al. Transcriptomic response of Chinese yew (Taxus chinensis) to cold stress. Front. Plant Sci. 8, 468 (2017).

11. Yuan, H. et al. Transcriptome profiles reveal cold acclimation and freezing tolerance of susceptible and tolerant hulless barley genotypes. Acta Physiol. Plant. 39, 275 (2017).

12. Zhang, L. L. \& Lin, Y. M. Tannins from Canarium album with potent antioxidant activity. J. Zhejiang Univ. Sci. B 9, 407-415 (2008).

13. Zeng, H. et al. Molecular structural characteristics of polysaccharide fractions from Canarium album (Lour.) Raeusch and their antioxidant activities. J. Food Sci. 80, H2585 (2015).

14. Xiang, Z., Wu, X. \& Liu, X. Chemical composition and antioxidant activity of petroleum ether extract of Canarium album. Pharm. Chem. J. 51, 606-611 (2017).

15. Zhang, L. L. \& Lin, Y. M. Determination of gallic acid content in extract of Canarium album (Lour.) Raeusch fruit and its radical scavenging activity. Chem. Ind. For. Prod. 15, 192-194 (2009).

16. Duan, W. et al. Isolation of anti-hiv components from Canarium album fruits by high-speed counter-current chromatography. Anal. Lett. 46, 1057-1068 (2013).

17. Xiang, Z. B., Mo, H. Q., Qu, Z. T., Xu, H. M. \& Jiang, H. Z. Ellagic acid from the dried fruits of Canarium album with antihepatitis B activity. Asian J. Chem. 23, 3759-3760 (2011).

18. Jia, Y. L. et al. Anti-tyrosinase kinetics and antibacterial process of caffeic acid N-nonyl ester in Chinese olive (Canarium album) postharvest. Int. J. Biol. Macromol. 91, 486-495 (2016).

19. Lai, R. L. et al. Advances on germplasm resource evaluation and cold resistance research in Chinese olive of China. Chinese J. Trop. Crops 38, 2188-2194 (2017) ((In Chinese)).

20. Shinozaki, Y. et al. High-resolution spatiotemporal transcriptome mapping of tomato fruit development and ripening. Nat. Commun. 9, 364 (2018).

21. Nasr, E. M. et al. Comparative transcriptome analysis of nodules of two mesorhizobium-chickpea associations with differential symbiotic efficiency under phosphate deficiency. Plant J. 91, 911-926 (2017).

22. Maruyama, K. et al. Identification of cold-inducible downstream genes of the Arabidopsis DREB1A/CBF3 transcriptional factor using two microarray systems. Plant J. 38, 982-993 (2010).

23. Zorina, A.A., Bedbenov, V.S., Novikova, G.V., Panichkin, V.B., Los', D.A. Involvement of serine/threonine protein kinases in the cold stress response in the cyanobacterium Synechocystis sp. PCC 6803: Functional characterization of SpkE protein kinase. Mol. Biol. 48, 452-462 (2014).

24. Wu, J., Zhang, Y., Yin, L., Qu, J. \& Lu, J. Linkage of cold acclimation and disease resistance through plant-pathogen interaction pathway in Vitis amurensis grapevine. Funct. Integr. Genomics 14, 741-755 (2014).

25. Zhang, C. \& Tian, S. Peach fruit acquired tolerance to low temperature stress by accumulation of linolenic acid and N-acylphosphatidylethanolamine in plasma membrane. Food Chem. 120, 864-872 (2010).

26. Powles, S. B., Berry, J. A. \& Bjorkman, O. Interaction between light intensity and chilling temperatures of inhibition of photosynthesis in chilling-sensitive plants. Plant Cell Environ. 6, 117-123 (2010).

27. Allen, D. J. \& Ort, D. R. Impacts of chilling temperatures on photosynthesis in warm-climate plants. Trends Plant Sci. 6, 36-42 (2001).

28. $\mathrm{Du}, \mathrm{H}$. et al. Carotenoid deficiency impairs ABA and IAA biosynthesis and differentially affects drought and cold tolerance in rice. Plant Mol. Biol. 83, 475-488 (2013).

29. Verma, V., Ravindran, P., Kumar, P. P. Plant hormone-mediated regulation of stress responses. BMC Plant Biol. 16, 86 (2016).

30. Chen, M. S. et al. Methyl jasmonate promotes phospholipid remodeling and jasmonic acid signaling to alleviate chilling injury in peach fruit. J. Agric. Food Chem. 67, 9958-9966 (2019).

31. Jaikumar, N. S., Snapp, S. S. \& Sharkey, T. D. Older Thinopyrum intermedium (Poaceae) plants exhibit superior photosynthetic tolerance to cold stress and greater increases in two photosynthetic enzymes under freezing stress compared with young plants. J. Exp. Bot. 67, 4743-4753 (2016).

32. Ciacka, K., Krasuska, U., Otulak-Kozieł, K. \& Gniazdowska, A. Dormancy removal by cold stratification increases glutathione and S-nitrosoglutathione content in apple seeds. Plant Physiol. Biochem. 138, 112-120 (2019).

33. Simon, F. et al. Physiological basis of chilling tolerance and early-season growth in miscanthus. Ann. Bot. 121, 281-295 (2018).

34. Dreyer, A. \& Dietz, K. J. Reactive oxygen species and the redox-regulatory network in cold stress acclimation. Antioxidants 7, 169 (2018).

35. Yang, T. \& Huang, X. S. Deep sequencing-based characterization of transcriptome of Pyrus ussuriensis in response to cold stress. Gene 661, 109-118 (2018).

36. Liu, S. et al. De novo transcriptome sequencing in Passiflora edulis Sims to identify genes and signaling pathways involved in cold tolerance. Forests 8, 435 (2017).

37. Yang, F. et al. Low-temperature-induced changes in the transcriptome reveal a major role of CgSVP genes in regulating flowering of Cymbidium goeringii. BMC Genomics 20, 53 (2019).

38. Wang, M. Q. et al. Transcriptomic analysis of Verbena bonariensis leaves under low-temperature stress. DNA Cell Biol. 38, 12331248 (2019).

39. Zhang, Y., Zhang, Y., Lin, Y., Luo, Y., Wang, X. R., Chen, Q., Sun, B., Wang, Y., Li, M. Y. \& Tang, H. A transcriptomic analysis reveals diverse regulatory networks that respond to cold stress in strawberry (Fragaria $\times$ ananassa). Int. J. Genomics 7106092 (2019).

40. Jain, M. \& Khurana, J. P. Transcript profiling reveals diverse roles of auxin-responsive genes during reproductive development and abiotic stress in rice. FEBS J. 276, 3148-3162 (2009).

41. Hannah, M. A., Heyer, A. G. \& Hincha, D. K. A global survey of gene regulation during cold acclimation in Arabidopsis thaliana. PLoS Genet. 1, e26 (2005).

42. Singh, V. K. \& Mukesh J. Genome-wide survey and comprehensive expression profiling of Aux/IAA gene family in chickpea and soybean. Front. Plant Sci. 6, 918 (2005).

43. Du, H. et al. A GH3 family member, OsGH3-2, modulates auxin and abscisic acid levels and differentially affects drought and cold tolerance in rice. J. Exp. Bot. 63, 6467-6480 (2012).

44. Hu, W. et al. Genome-wide analysis of poplar SAUR gene family and expression profiles under cold, polyethylene glycol and indole-3-acetic acid treatments. Plant Physiol. Biochem. 128, 50-65 (2018).

45. Oh, W., Kim, J., Kim, Y. H., Lee, I. J. \& Kim, K. S. Shoot elongation and gibberellin contents in Cyclamen persicumare influenced by temperature and light intensity. Hortic. Environ. Biotechnol. 56, 762-768 (2015).

46. Hisamatsu, T., Koshioka, M. Cold treatments enhance responsiveness to gibberellin in stock (Matthiola incana (L.) R. Br.). J. Horticult. Sci. Biotechnol. 75, 672-678 (2000).

47. Lee, S. et al. Gibberellin regulates Arabidopsis seed germination via RGL2, a GAI/RGA-like gene whose expression is up-regulated following imbibition. Genes Dev. 16, 646-658 (2002).

48. Achard, P. Integration of plant responses to environmentally activated phytohormonal signals. Science 311, 91-94 (2006). 
49. Wang, F. et al. Crosstalk of PIF4 and DELLA modulates CBF transcript and hormone homeostasis in cold response in tomato. Plant Biotechnol. J. 18, 1041-1055 (2020).

50. Yoshida, T., Mogami, J. \& Yamaguchi-Shinozaki, K. ABA-dependent and ABA-independent signaling in response to osmotic stress in plants. Curr. Opin. Plant Biol. 21, 133-139 (2014).

51. Zhang, H. et al. Cloning and characterization of NtSnRK2.7 and NtSnRK2.8 genes involved in abiotic stress responses from Nicotiana tabacum. Acta Physiol. Plant. 36, 1673-1682 (2014).

52. Zhang, H., Mao, X. \& Jing, R. SnRK2 acts within an intricate network that links sucrose metabolic and stress signaling in wheat. Plant Signal. Behav. 6, 652-654 (2001).

53. Mao, X., Zhang, H., Tian, S., Chang, X. \& Jing, R. TaSnRK2.4, an SNF1-type serine/threonine protein kinase of wheat (Triticum aestivum L.), confers enhanced multistress tolerance in Arabidopsis. J. Exp. Bot. 61, 683-696 (2010).

54. Tähtiharju, S. \& Palva, T. Antisense inhibition of protein phosphatase $2 \mathrm{C}$ accelerates cold acclimation in Arabidopsis thaliana. Plant J. 26, 461-470 (2002).

55. Wasternack, C. \& Hause, B. Jasmonates: Biosynthesis, perception, signal transduction and action in plant stress response, growth and development. Ann. Bot. 111, 1021-1058 (2013).

56. Liu, W. et al. Cold stress improves the production of artemisinin depending on the increase of endogenous jasmonate. Biotechnol. Appl. Biochem. 64, 305-314 (2017).

57. Yin, X. R. et al. Ethylene-related genes show a differential response to low temperature during 'Hayward' kiwifruit ripening. Postharvest Biol. Technol. 52, 9-15 (2009).

58. Zou, Y. et al. The relationship between the expression of ethylene-related genes and papaya fruit ripening disorder caused by chilling injury. PLoS ONE 9, el16002 (2014).

59. Gatz, C. From pioneers to team players: TGA transcription factors provide a molecular link between different stress pathways. Mol. Plant Microbe Interact. 26, 151-159 (2013).

60. Goyal, R. K. et al. Pathogenesis-related protein $1 \mathrm{bl}$ (PR1b1) is a major tomato fruit protein responsive to chilling temperature and upregulated in high polyamine transgenic genotypes. Front. Plant Sci. 7, 901 (2016).

61. Grabherr, M. G. et al. Full-length transcriptome assembly from RNA-Seq data without a reference genome. Nat. Biotechnol. 29, $644(2011)$.

62. Pei, M., Niu, J., Li, C., Gao, F. \& Quan, S. Identification and expression analysis of genes related to calyx persistence in Korla fragrant pear. BMC Genomics 17, 132 (2016).

63. Wang, L., Feng, Z., Wang, X., Wang, X. \& Zhang, X. DEGseq: An R package for identifying differentially expressed genes from RNA-seq data. Bioinformatics 26, 136-138 (2010).

64. Storey, J. D. \& Tibshirani, R. Statistical significance for genomewide studies. Proc. Natl. Acad. Sci. 100, 9440-9445 (2003).

65. Young, M. D., Wakefield, M. J., Smyth, G. K. \& Oshlack, A. Gene ontology analysis for RNA-seq: Accounting for selection bias. Genome Biol. 11, R14 (2010).

66. Mao, X., Tao, C. J. G. O. \& Wei, L. Automated genome annotation and pathway identification using the KEGG Orthology (KO) as a controlled vocabulary. Bioinformatics 21, 3787-3793 (2005).

67. Kanehisa, M. \& Goto, S. KEGG: Kyoto encyclopedia of genes and genomes. Nucleic Acids Res. 28, 27-30 (2000).

68. Huang, M.J. Measurement of Hyperoside Content, Cloning and Expression of the Synthesis Genes in Canarium album. (Fujian Agriculture and Forestry University, 2017).

\section{Acknowledgements}

This research was supported by the Natural Science Foundation of Fujian Province of China (2019J01110), the Basic Sci-tech Project of Provincial Public Welfare Scientific Research Institution of Fujian Province of China (2018R1013-3) and the Project of Resource Protection of Species and Varieties (Tropical Crops) of Ministry of Agriculture and Rural Affairs of China (151821301354051701).

\section{Author contributions}

R.L.L., R.J.W., C.Z.C. and X.F. performed most of the experiments and designed the study; R.L.L., C.Z.C. and R.J.W. wrote the manuscript; J.C. and X.X.W. collected samples and performed the pre-experiments; R.L.L, C.Z.C and Y.T.C. performed most of the bioinformatics studies. All authors reviewed the manuscript.

\section{Competing interests}

The authors declare no competing interests.

\section{Additional information}

Correspondence and requests for materials should be addressed to C.C. or R.W.

Reprints and permissions information is available at www.nature.com/reprints.

Publisher's note Springer Nature remains neutral with regard to jurisdictional claims in published maps and institutional affiliations.

Open Access This article is licensed under a Creative Commons Attribution 4.0 International License, which permits use, sharing, adaptation, distribution and reproduction in any medium or format, as long as you give appropriate credit to the original author(s) and the source, provide a link to the Creative Commons licence, and indicate if changes were made. The images or other third party material in this article are included in the article's Creative Commons licence, unless indicated otherwise in a credit line to the material. If material is not included in the article's Creative Commons licence and your intended use is not permitted by statutory regulation or exceeds the permitted use, you will need to obtain permission directly from the copyright holder. To view a copy of this licence, visit http://creativecommons.org/licenses/by/4.0/.

(C) The Author(s) 2021 